\title{
Transcriptome sequencing and whole genome expression profiling of hexaploid sweetpotato under salt stress
}

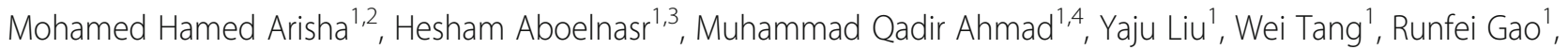
Hui Yan ${ }^{1}$, Meng Kou ${ }^{1}$ Xin Wang ${ }^{1}$, Yungang Zhang ${ }^{1}$ and Qiang Li ${ }^{1 *}$

\begin{abstract}
Background: Purple-fleshed sweetpotato (PFSP) is one of the most important crops in the word which helps to bridge the food gap and contribute to solve the malnutrition problem especially in developing countries. Salt stress is seriously limiting its production and distribution. Due to lacking of reference genome, transcriptome sequencing is offering a rapid approach for crop improvement with promising agronomic traits and stress adaptability.

Results: Five cDNA libraries were prepared from the third true leaf of hexaploid sweetpotato at seedlings stage (Xuzi-8 cultivar) treated with $200 \mathrm{mM} \mathrm{NaCl}$ for 0, 1, 6, 12, 48 h. Using second and third generation technology, Illumina sequencing generated 170,344,392 clean high-quality long reads that were assembled into 15,998 unigenes with an average length 2178 base pair and $96.55 \%$ of these unigenes were functionally annotated in the NR protein database. A number of 537 unigenes failed to hit any homologs which may be considered as novel genes. The current results indicated that sweetpotato plants behavior during the first hour of salt stress was different than the other three time points. Furthermore, expression profiling analysis identified 4, 479, 281, 508 significantly expressed unigenes in salt stress treated samples at the different time points including 1, 6, 12, $48 \mathrm{~h}$, respectively as compared to control. In addition, there were 4, 1202, 764 and 2195 transcription factors differentially regulated DEGs by salt stress at different time points including 1, 6, 12, 48 h of salt stress. Validation experiment was done using 6 randomly selected unigenes and the results was in agree with the DEG results. Protein kinases include many genes which were found to play a vital role in phosphorylation process and act as a signal transductor/ receptor proteins in membranes. These findings suggest that salt stress tolerance in hexaploid sweetpotato plants may be mainly affected by TFs, PKs, Protein Detox and hormones related genes which contribute to enhance salt tolerance.
\end{abstract}

Conclusion: These transcriptome sequencing data of hexaploid sweetpotato under salt stress conditions can provide a valuable resource for sweetpotato breeding research and focus on novel insights into hexaploid sweetpotato responses to salt stress. In addition, it offers new candidate genes or markers that can be used as a guide to the future studies attempting to breed salt tolerance sweetpotato cultivars.

Keywords: Hexaploid sweetpotato, Salt stress, Expression profile, RNA-sequencing, Transcriptome

\footnotetext{
*Correspondence: instrong@163.com

${ }^{1}$ Xuzhou Institute of Agricultural Sciences in Jiangsu Xuhuai District / Key

Laboratory of Biology and Genetic Improvement of Sweetpotato, Ministry of

Agriculture / Sweetpotato Research Institute, CAAS, Xuzhou 221131, Jiangsu,

China

Full list of author information is available at the end of the article
}

(c) The Author(s). 2020 Open Access This article is distributed under the terms of the Creative Commons Attribution 4.0 International License (http://creativecommons.org/licenses/by/4.0/), which permits unrestricted use, distribution, and reproduction in any medium, provided you give appropriate credit to the original author(s) and the source, provide a link to the Creative Commons license, and indicate if changes were made. The Creative Commons Public Domain Dedication waiver (http://creativecommons.org/publicdomain/zero/1.0/) applies to the data made available in this article, unless otherwise stated. 


\section{Background}

Sweetpotato (Ipomoea batatas (L.) Lam.), the only crop plant belongs to Convolvulaceae family with starchy storage roots. Purple-fleshed sweetpotato (PFSP) considered to be an important source for anthocyanin which displays strong antioxidant properties [1]. It is also considered as an important staple source of calories and proteins which consumed by all age groups. In terms of agricultural production sweetpotato considered as the seventh most important food crop in the world [2].

Salinity is a global problem caused vast area of lands remaining uncultivated. Exposure of sweetpotato plants to salt stress resulting in problems such as ion imbalance, mineral deficiency, osmotic stress, ion toxicity and oxidative stress [3]. Ultimately, these conditions interact with several cellular components including DNA, protein, lipids and pigments. That's in rule impeding plant development and affect sweetpotato production [4]. Therefore, introducing of salt tolerant sweetpotato cultivar became necessary.

With the fact of environmental stress and climate change there is an urgent need to accelerate crops breeding with higher production and stress tolerance traits [5]. In sweetpotato transcriptome sequencing offers a rapid approach for crop improvement with promising agronomic traits and stress adaptability. Several transcriptome sequencing studies have been conducted on hexaploid sweetpotato genome [6-8]. However, having a complex genome structures $(2 n=6 x=90)$, sweetpotato still didn't achieve a reference genome which covered a few percent of genome, so still a long way from the reference genome [9].

Currently, referring to the potential advantages of anthocyanin for health, more attention was paid to transcriptome analysis of purple flesh sweetpotato [10]. Most of conducted transcriptome sequencing on PFSP focused on genes related to anthocyanins biosynthesis and their regulation mechanism $[11,12]$. While, few researches have been done on the effect of biotic or abiotic stress on PFSP.

In the present study, second and third generation sequencing technology were used to establish a useful database of transcriptomes sequencing as well as differentially expressed genes in sweetpotato leaves under salt stress conditions. In total $102,845,433$ high quality reads were assembled into 16,856 transcripts giving 15,998 unigenes. Our results provide novel insights into hexaploid sweetpotato response to salt stress and identified numerous specific genes involved in salt stress defense mechanisms. That's in role can be used to guide future efforts towards breeding of sweetpotato salt resistant cultivars.

\section{Results}

Sequencing and de novo assembly of sweetpotato transcriptome under salt stress conditions

For NGS, five cDNA libraries were prepared from the third true leaf of PFSP seedlings (Xuzi-8 cultivar) treated with $200 \mathrm{mM} \mathrm{NaCl}$ for $0,1,6,12,48 \mathrm{~h}$. These libraries were separately sequenced using Illumina high-throughput second generation sequencing platform. After removing the low-quality reads and all possible contaminations, a total of 170,344,392 clean reads with Q20>96.73\% and GC percentage between 45.07 and $46.50 \%$ were used for further study (Table 1). Each library was represented by over than 30 million high-quality reads, with number ranging from $32,830,183$ to $35,663,873$. For 3rd GS, four time points RNA samples including1, 6, 12 and $48 \mathrm{~h}$ were mixed to produce one library beside to the control library. These libraries were separately sequenced using Illumina highthroughput third generation sequencing platform. TPM, FPKM, RPKM and fold change (FC) were recorded for each replicate of each library separately on both NGS and 3rd GS. Obtained sequence from NGS and 3rd GS were aligned and similar sequence data from all libraries/samples were pooled. Due to the lack of a reference genome, the clean reads resulted in from the transcriptome sequences were aligned and assembled using Trinity software. After further clustering and assembly, a total of 21,497,466; 20,272,643; $21,954,725 ; 19,121,890$ and 19,998,709 mapped reads were obtained with percentage $60.26,61.79,61.62,59.33$ and $58.87 \%$ of total reads at different time points $(0,1,6,12,48$ h), respectively. As shown in Table 1 that the average length of transcripts and unigenes was more than $2000 \mathrm{bp}$ which indicate that the obtained data are high quality data. Statistics on unigenes and transcripts length resulted from mixed second and third generations sequencing were performed using PacBio's officially recommended cogent software (Tables 1 and 2, Fig. 1). In addition, the total number of CDS was 30,615 of which 23,245 CDS mapped to the protein database.

\section{Functional annotation}

To annotate the obtained unigenes, a BlastX search against the NR NCBI protein database with cut-off Evalue of $10^{-5}$ based on sequence similarity was performed. In total, 15,461 unigenes were detected (Table 3) that showed comparability with known gene sequence in all databases corresponding to approximately $96.64 \%$ of total unigenes including Clusters of orthologous groups (COG), Gene ontology (GO), Kyoto encyclopaedia of genes and genomes (KEGG), eukaryotic orthologous group (KOG), protein family (PFAM), Swiss-Prot., NCBI non-redundant protein sequences $(\mathrm{Nr})$. According to Fig. 1b, the species that gave the best BlastX matches were Nicotiana sylvestris (21.30\%) followed by Nicotiana tomentosiformis (20.69\%), Solanum tuberosum (9.16\%), Sesamum indicum (7.46\%), Coffea canephora (6.00\%), Solanum lycopersicum (4.74\%), Solanum penellii (4.58\%), Ipomoea batatas (3.07\%), Vitis vinifera (2\%), Ipomoea nil (1.57\%) and others (19.22\%). 
Table 1 Next generation sequencing statistical summary of sequenced and assembled results

\begin{tabular}{llllll}
\hline & $0 \mathrm{~h}$ & $1 \mathrm{~h}$ & $6 \mathrm{~h}$ & $12 \mathrm{~h}$ & $48 \mathrm{~h}$ \\
\hline Total Reads & $35,663,873$ & $32,830,183$ & $35,632,937$ & $32,241,116$ & $33,976,283$ \\
Mapped Reads & $21,497,466$ & $20,272,643$ & $21,954,725$ & $19,121,890$ & $19,998,709$ \\
Mapped Ratio & $60.26 \%$ & $61.79 \%$ & $61.62 \%$ & $59.33 \%$ & $58.87 \%$ \\
Nt & $10,699,162,000$ & $9,849,054,900$ & $10,689,881,200$ & $9,672,334,900$ & $10,192,884,900$ \\
GC (\%) & 45.53 & 46.50 & 46.17 & 45.29 & 45.07 \\
Q20 (\%) & 96.72 & 96.63 & 96.77 & 96.75 & 96.77 \\
Q30 (\%) & 92.03 & 91.66 & 92.08 & 91.91 & 91.96 \\
N Percentage & 00.00 & 00.00 & 00.00 & 00.00 & 00.00 \\
\hline
\end{tabular}

Note: Nt, total number of clean nucleotides; The GC percentage is the proportion of guanidine and cytosine nucleotides among total nucleotides; The Q20 and Q30 percentage is the proportion of nucleotides with a quality value $>20$ and 30 , respectively; The $\mathrm{N}$ percentage is the proportion of unknown nucleotides in clean reads

\section{Gene ontology (GO) and KOG classifications}

For functional categories of 15,998 successfully annotated unigenes, a total of 12,481 genes (78.01\%) (Table 3 and Additional file 1) were assigned to at least one GO term. These GO terms were categorized into 48 functional groups which were divided into three categories including biological process, cellular component and molecular function (Fig. 2 and Additional file 2). For biological process, the highest categories were metabolic (8291 unigenes, $53.55 \%$ ) followed by cellular process (7774, 50.21\%) then single organism process (6181, $39.92 \%)$. In the category of molecular function, the most abundant groups included catalytic activity (6369, $41.14 \%)$ and binding activity (6513, 42.07\%). Furthermore, the most abundant group for cellular components was cell parts $(7198,46.49 \%)$ (Additional file 3).

Genetic orthologous relationships, combines evolutionary relationships were used to classify the potential functions into different orthologous clusters (COG). In

Table 2 Third generation sequencing statistical summary of sequenced and assembled results

\begin{tabular}{lll}
\hline & Unigenes & Transcripts \\
\hline Total number of sequences & 15,998 & 16,856 \\
Total sequences length & $34,848,832$ & $36,928,928$ \\
Maximum length & 9,135 & 9,135 \\
Minimum length & 208 & 208 \\
Average length & 2,178 & 2,190 \\
Percent GC & $43.10 \%$ & $43.12 \%$ \\
N40 & 3,698 & 3,733 \\
N50 & 2,652 & 2,701 \\
N60 & 2,131 & 2,155 \\
N70 & 1,785 & 1,795 \\
N80 & 1,503 & 1,509 \\
N90 & 1,162 & 1,168 \\
\hline
\end{tabular}

N50, represents sorting the assembled transcripts from long to short by length, accumulating the length of the transcript to $50 \%$ of the total length, corresponding to the length of the transcript, and so on total 10,020 genes were subdivided into 25 functional classes as shown in Fig. 3 and Additional file 1. Among the 25 groups, "general function prediction only" represented the largest group (1871 unigenes, 16.89\%) followed by "post translational modification, protein turnover, chaperons" (1271 unigenes, 11.47\%) then "signal transduction mechanisms" (1037 unigenes, 9.36\%). In addition, it was interesting to note that 87 genes were aligned to the "defense mechanisms" cluster (Fig. 3). Rigorous algorithm (FDR $\leq 0.001, \log 2$ FC-ratio $\geq 1$ ) were applied to measure the significance level of the 87 obtained genes. Out of these defense mechanisms genes there were no significant expressed genes during the first hour of salt stress. Furthermore, there were two unigenes, (unigene802 and 1120) which significantly upregulated during 6,12 and $48 \mathrm{~h}$ of salt stress, these two unigenes were aligned to GID1-like gibberellin receptor. On the other hand the unigene6088 (cinnamoyl-Co-A reductase 1 - like) was significantly down-regulated at $6 \mathrm{~h}$ of salt stress. In addition, at $48 \mathrm{~h}$ of salt stress, there were two unigenes (5647 and 5851) significantly downregulated which were aligned to Alpha/beta hydrolase (carboxylesterase) and cinnamoyl-CoA reductase 1-like (Additional file 1).

\section{KEGG annotations}

KEGG pathway annotation for 15,461 unigenes was obtained as shown in Fig. 4. A total of 5965 sequences were assigned to 125 pathways. The largest enriched groups in the KEGG pathways were "Metabolic pathways (ko01100)" (1508 unigenes, 25.28\%) and "Biosynthesis of secondary metabolites (ko01110)" (733 unigenes, 12.28\%), which ranked at 1st position. Followed by "Carbon metabolism (ko01200)" (336 unigenes, 5.63\%), "Ribosome (ko03010)" (303 unigenes, 5.08\%), "Plant hormone signal transduction (ko04075)" (249 unigenes, 4.17\%), "Biosynthesis of amino acids (ko01230)" (249 unigenes, 4.17\%) and "Photosynthesis (ko00195)" (199 unigenes, 3.34\%). These specific enrichments KEGG pathways and mechanisms are 


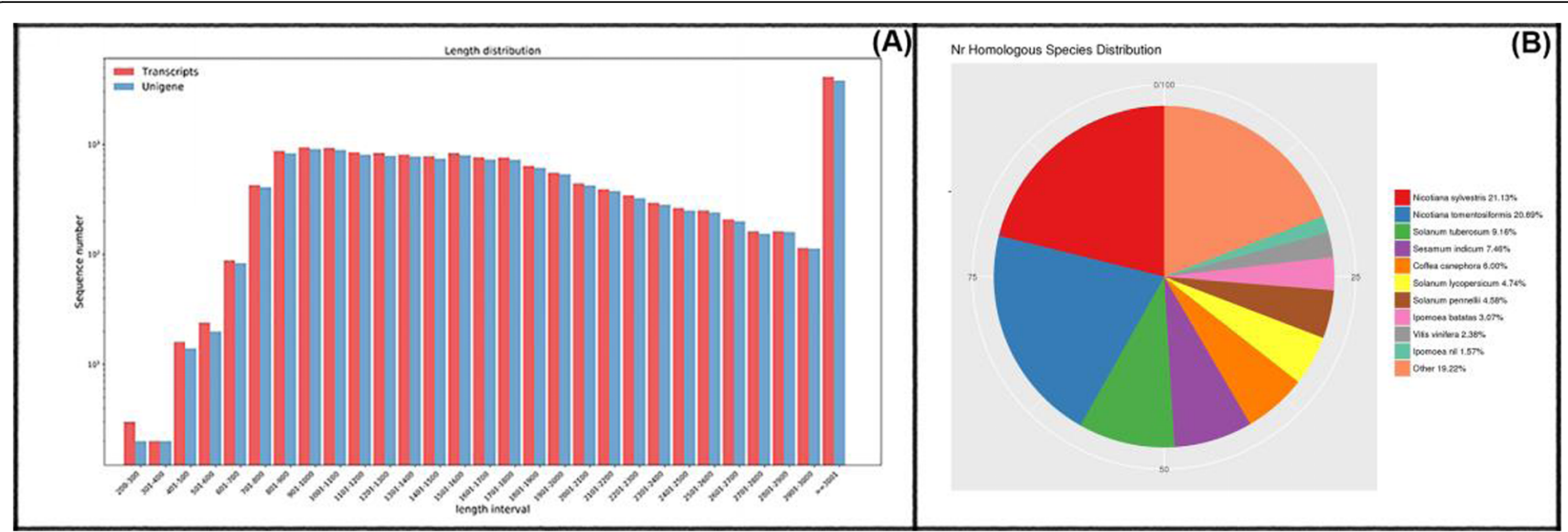

Fig. 1 a Assembly result sequence length distribution map of transcripts and unigenes in Xuzi-8 sweetpotato cultivar. The horizontal axis represents the length intervals of the transcripts and unigenes, and the vertical axis represents the number of transcripts and unigenes. $\mathbf{b}$ Species distribution of the top BlastX matches of the transcriptome unigenes of Xuzi-8 sweetpotato cultivar in the non-redundant protein database (Nr) data base

involved in response to salt stress in sweetpotato (Xuzi-8 cultivar) (Additional file 4).

\section{Expression patterns of hexaploid sweetpotato unigenes in response to salt stress}

The results in Fig. 5 (a-d) showed the phenotypic changes during salt stress exposure as compared to control. Salt stress visual symptoms in the form of welting started slightly at $12 \mathrm{~h}$ and increased gradually showing slight leaves folding at $48 \mathrm{~h}$. The highest number of DEGs was induced at $48 \mathrm{~h}$ of salt stress followed by $6 \mathrm{~h}$ and $12 \mathrm{~h}$, respectively, while $1 \mathrm{~h}$ gave the lowest number of DEGs. Transcriptional level at $1,6,12,48 \mathrm{~h}$ as compared to control induced expression values 4, 529, 341 and 663 as up-regulated unigenes, and 0, 672, 422 and 1531 as downregulated, respectively. Furthermore, there were 15,534; 14,$450 ; 14,703$ and 13,330 normally expressed unigenes during 1, 6, 12 and $48 \mathrm{~h}$ of salt stress. In addition, there were 119 up-regulated genes, 87 down-regulated genes, 12,384 genes normal and 211 unknown genes common under all durations of salt stress (Fig. 5 e-h).
Detection of salt-induced genes related to salt tolerance RPKM read counts were used to identify DEGs significance level between control and salt-stressed samples using the rigorous algorithm (FDR $\leq 0.001, \log 2$ FCratio $\geq 1$ ) for significantly up-regulated unigenes and $(F D R \leq 0.001, \log 2$ FC-ratio $\leq-1)$ for significantly down regulated genes. Furthermore, number of 4, 479, 281, 508 unigenes were up-regulated with significant expression level in salt stress treated samples at the different time points of salt stress including $1,6,12,48 \mathrm{~h}$, respectively. On the other hand, there were 567, 301, 1335 unigenes significantly down-regulated at $6,12,48 \mathrm{~h}$ of salt stress (Fig. 7).

During the first hour of salt stress there were four significantly expressed unigenes including SBP-domain, HSP-70, pectin methyl esterase inhibitor, and uncharacterized protein sequence gene families, respectively (Fig. 6 and Table 4).

After $6 \mathrm{~h}$ a number of 479 unigenes were significantly up-regulated, these genes belong to 45 different protein families and most of these families are involved in stress tolerance or defense mechanisms and metabolism, etc.

Table 3 Statistics of unigenes annotated in public database

\begin{tabular}{llll}
\hline Annotated Database & $\begin{array}{l}\text { Annotated Number } \\
\text { Value (\%) }\end{array}$ & $\begin{array}{l}300<=\text { length<1000 } \\
\text { Value (\%) }\end{array}$ & $\begin{array}{l}\text { length>=1000 } \\
\text { Value (\%) }\end{array}$ \\
\hline COG & $6982(43.64 \%)$ & $853(12.22 \%)$ & $6129(87.78 \%)$ \\
GO & $12480(78.01 \%)$ & $1750(14.02 \%)$ & $10728(85.96 \%)$ \\
KEGG & $5965(37.29 \%)$ & $806(13.51 \%)$ & $5159(86.49 \%)$ \\
KOG & $10020(62.63 \%)$ & $1164(11.62 \%)$ & $8856(88.38 \%)$ \\
Pfam & $13569(84.82 \%)$ & $1725(12.71 \%)$ & $11844(87.29 \%)$ \\
Swiss-Prot. & $13428(83.94 \%)$ & $1675(12.47 \%)$ & $11751(87.51 \%)$ \\
Nr & $15446(96.55 \%)$ & $2115(13.69 \%)$ & $13329(86.29 \%)$ \\
All & $15461(96.64 \%)$ & $2125(13.74 \%)$ & $13334(86.24 \%)$ \\
\hline
\end{tabular}




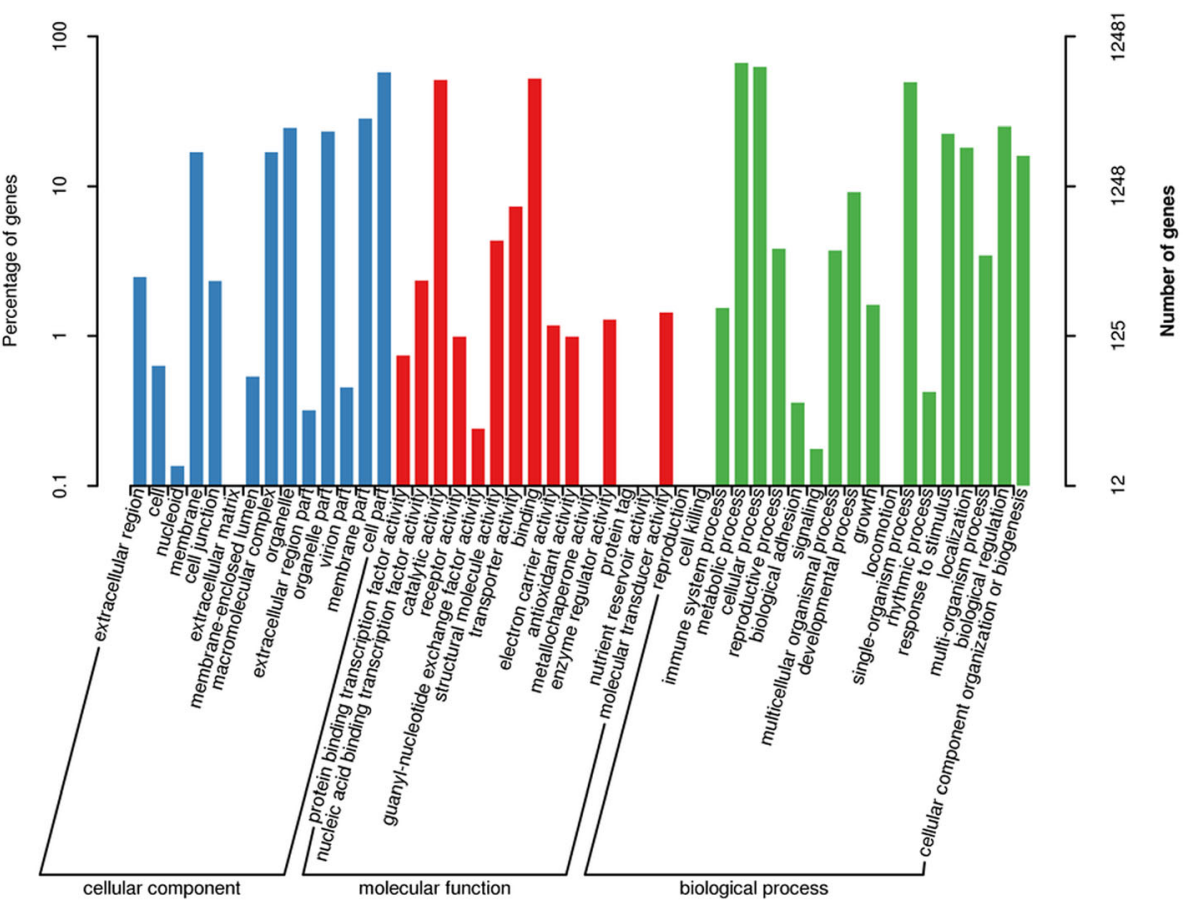

Fig. 2 Gene ontology (GO) classifications in sweetpotato (Xuzi-8 cultivar), the percentage indicate the proportion of unigenes with the GO annotations

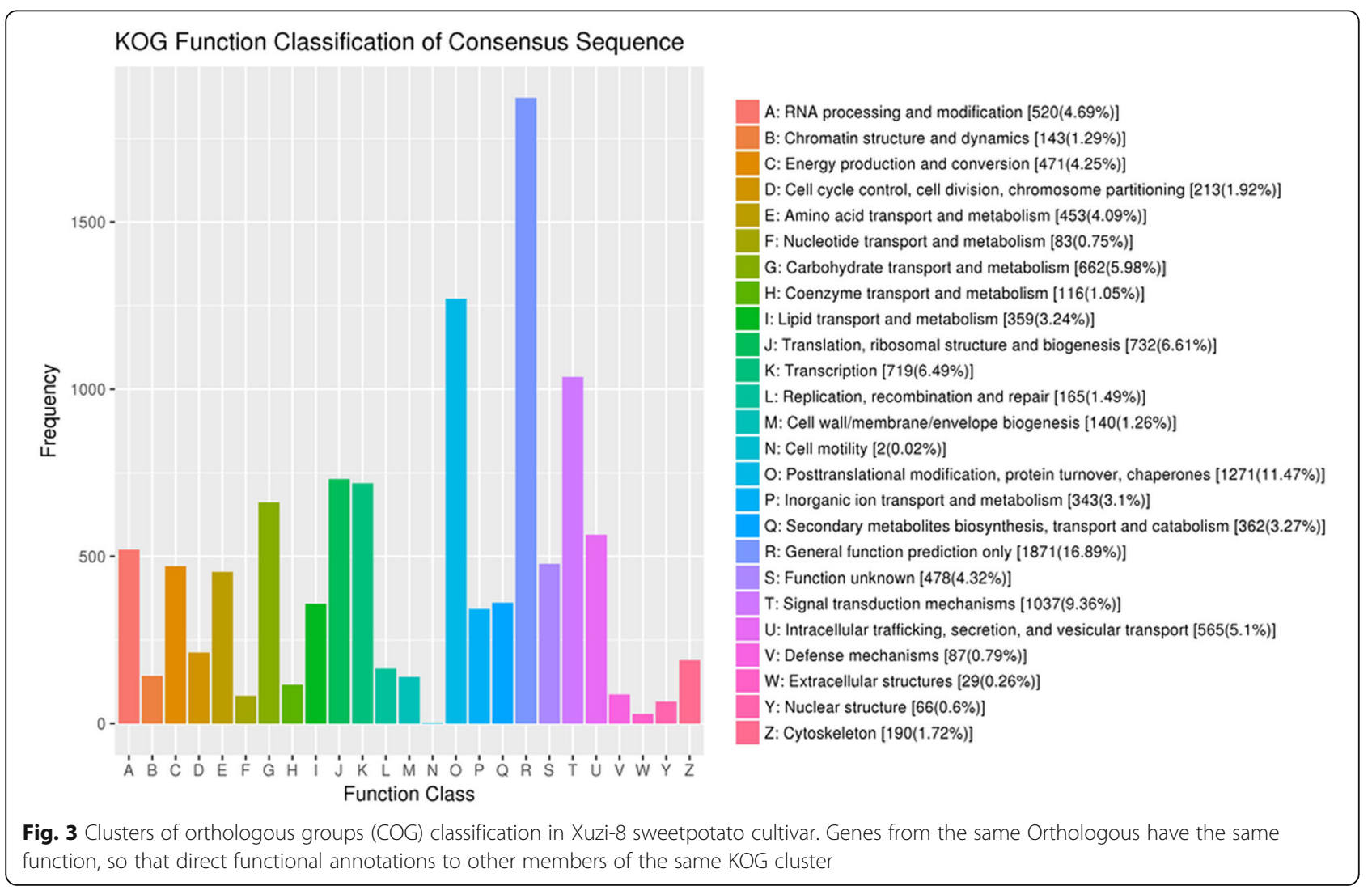




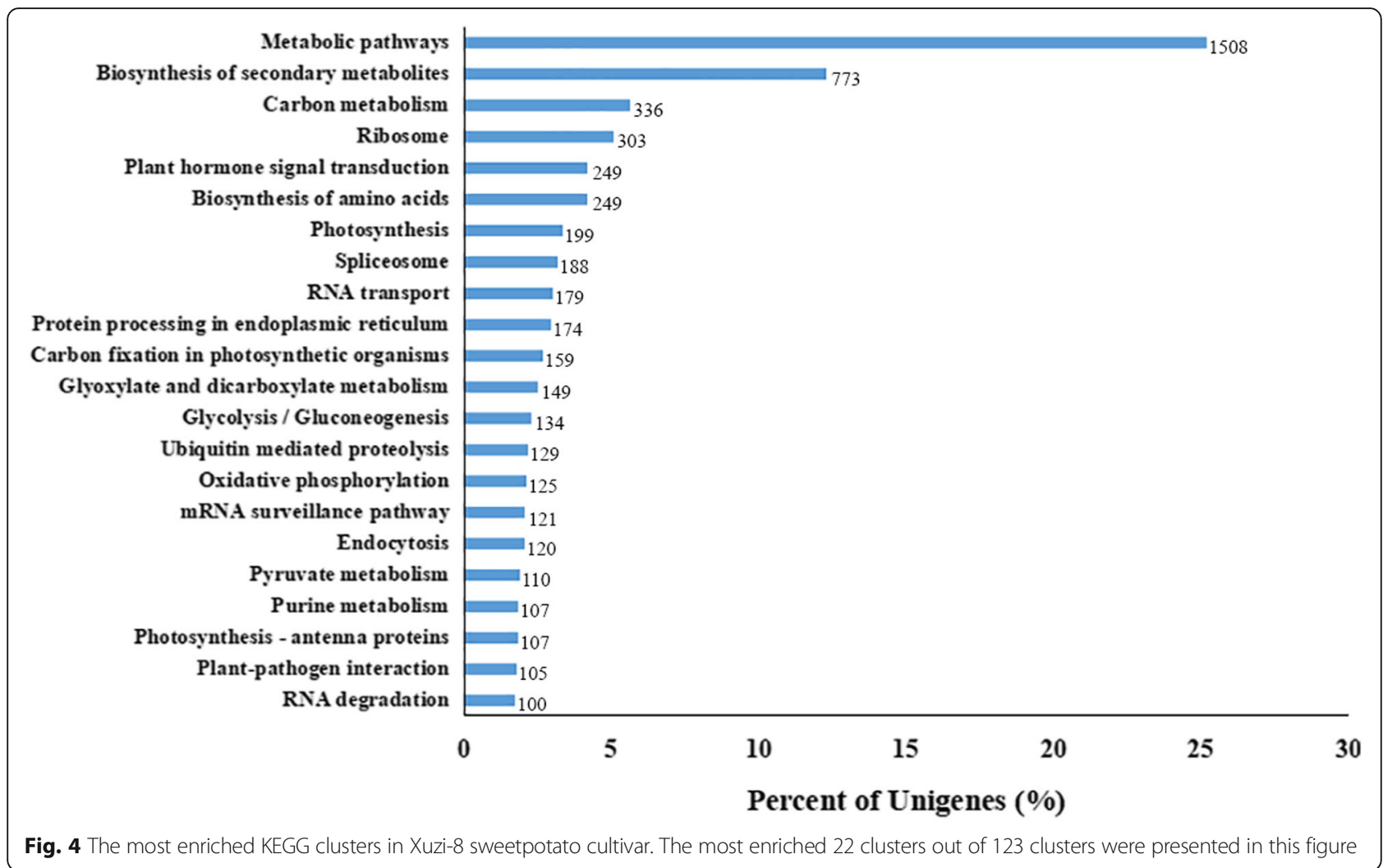

Fig. 4 The most enriched KEGG clusters in Xuzi-8 sweetpotato cultivar. The most enriched 22 clusters out of 123 clusters were presented in this figure

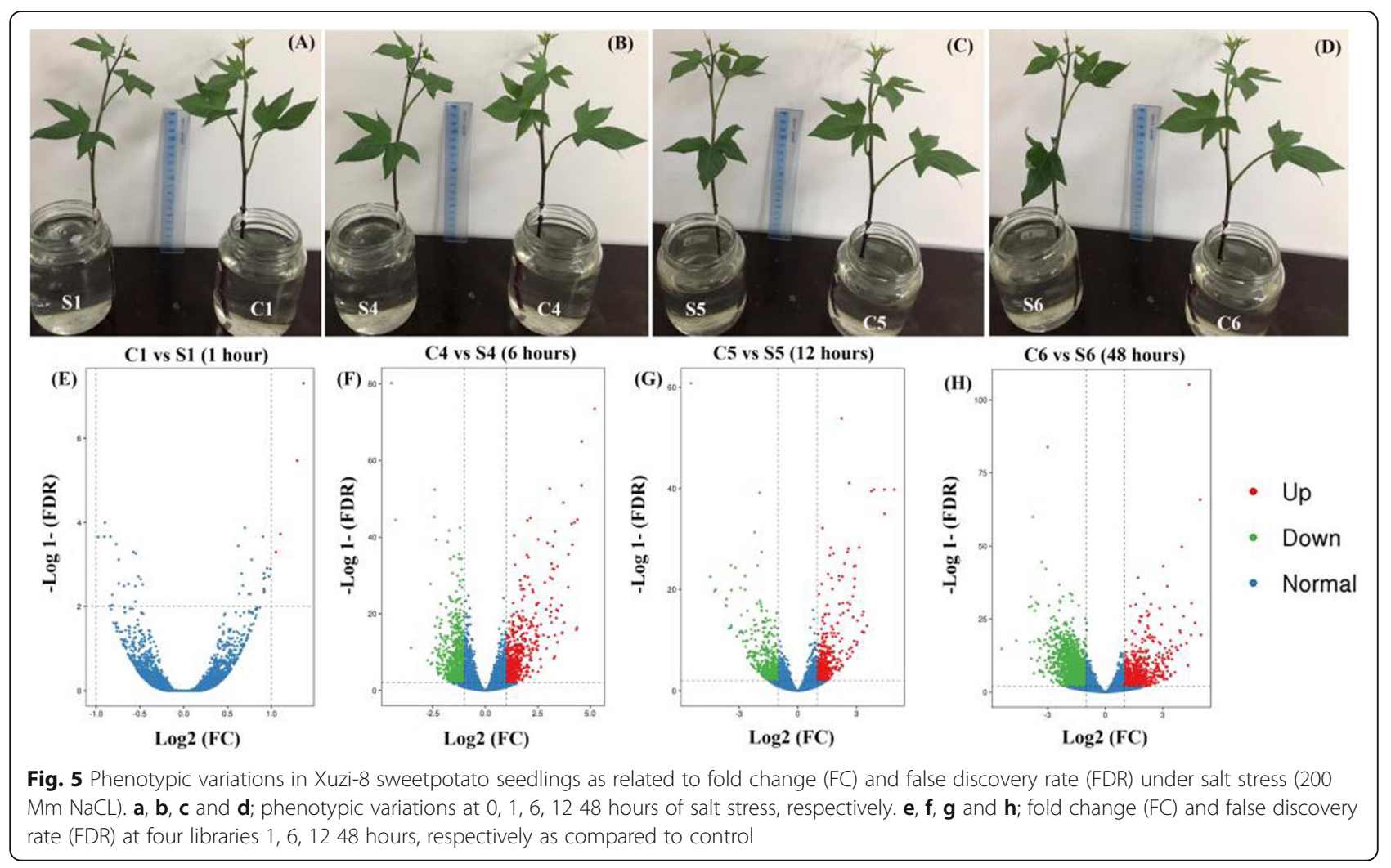




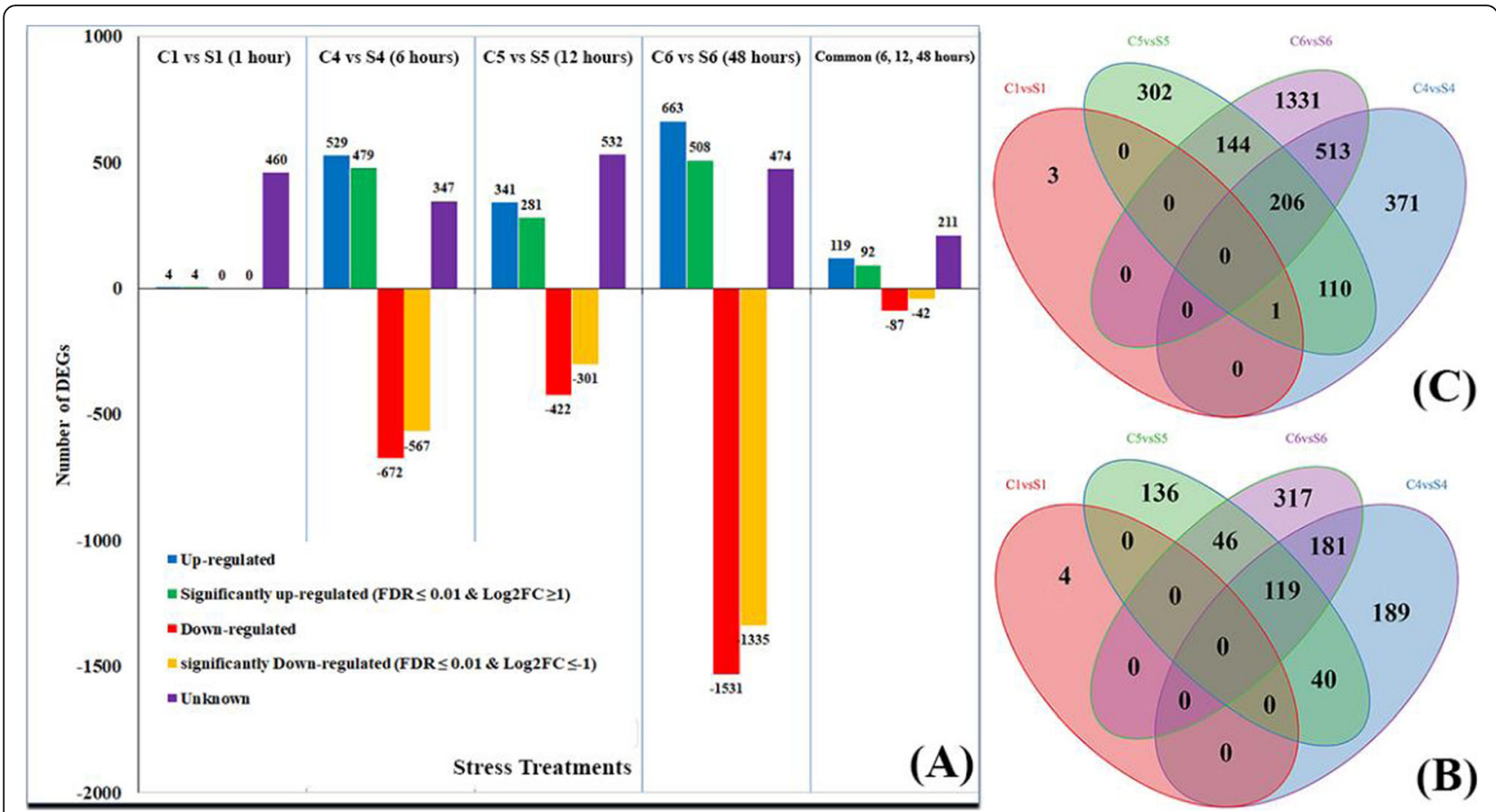

Fig. 6 Comparison of four transcriptomes for classification of DEGs and statistics of sequence annotation of DEGs. a; Statistical chart of DEGs transcriptome in response to salt stress. Transcriptional level of five libraries including 1, 6, 12 and 48 hours of salt stress treatment as compared to control. $\mathbf{b}$ and $\mathbf{c}$; Venn diagram analysis of up-regulated unigenes and all induced unigenes, respectively.

Between these 479 unigenes there were 5 unigenes directly related to salt stress including bZIP-8 transcription factor, EID1-like F-box protein-3, WCOR-413 like cold acclimation protein and putative low temperature and salt responsive protein isoform with fold change values 1.8, 1.9, 3.6, 1.6 and 2.1 higher than control. Furthermore, among all significantly expressed genes there were 9 genes which gave the highest expression level. These nine genes were included under three different protein families i.e., malate synthase, glyoxysomal; protein TRANSPARENT TESTA-12, detoxification; SNF-1 related protein kinase and two dehydrin unigenes (Table 4).

While, there were 567 unigenes significantly downregulated at $6 \mathrm{~h}$ of salt stress. Among these genes there were 15 salt stress response unigenes belonging to different protein families including plastid glutamine synthase, Cellulose synthase A-catalytic (UDP-forming), decarboxylase transporter-1 (chloroplast), Indole-3 acetic acid amino synthase (GH3), tubulin alpha-2 chain-like, protein WALLSARE THIN-1 like and aquaporin protein-12. The highest down regulated unigenes (4 folds lower than control) were two unigenes belong to nitrate reductase (NADH) protein family (Table 4).

At $12 \mathrm{~h}$ of salt stress, a number of 281 unigenes were significantly expressed and up-regulated which belong to 32 different gene families. Between these genes there were 5 genes directly respond to salt stress treatment which were aligned to BEL-1 like homes domain protein-1 (BLH-1), bZIP-8 transcription factor and EID1 like F-box protein-3. Moreover, the superior expressed genes were belonged to nucleoredoxin2 isoform X-1 (AhpC/TSA gene family). On the other hand, a number of 301 unigenes were significantly down-regulated including six unigenes involved in response to salt stress. These 6 unigenes belong to four different gene families i.e., cellulose synthase A catalytic (UDP forming), Tubulin alpha- 2 chain-like isoform $\times 2$ and enol-[acyl-carrier-protein] reductase (NADH)- chloroplast-like. Furthermore, four unigenes were expressed four folds higher than control which included under four different gene families (elongation factor [TSF], ACT-domain containing protein [ACR-11], proline rich protein and protein like isoform and pectin methyl-esterase inhibitor (Table 4).

There were 508 unigenes significantly up-regulated in leaf tissues after $48 \mathrm{~h}$ of salt stress belonging to 63 different gene families. The unigenes which responded to salt stress were included under Cystein-rich receptor-like protein kinase-2, bZIP-8 transcription factor, EID-1 like F-box protein- 3 and low temperature and salt responsive protein isoform. Furthermore, the highest expressed genes in leave tissues were belong to importin- 5 malate synthase, glyoxysomal, protein phosphatase 2C-37 like, O-acyltransferase WSD-1-like, EID1-like F-box protein-3 and $\mathrm{NADH}$-dehydrogenase. In addition there were 1335 
Table 4 Salt stress defence mechanism related genes, Genes involved in physiological processes related to response to salt stress and highest significantly expressed genes that were significantly up and down regulated under different durations $(1,6,12,48$ hours) of salt stress (200 mM NaCl) as compared to control

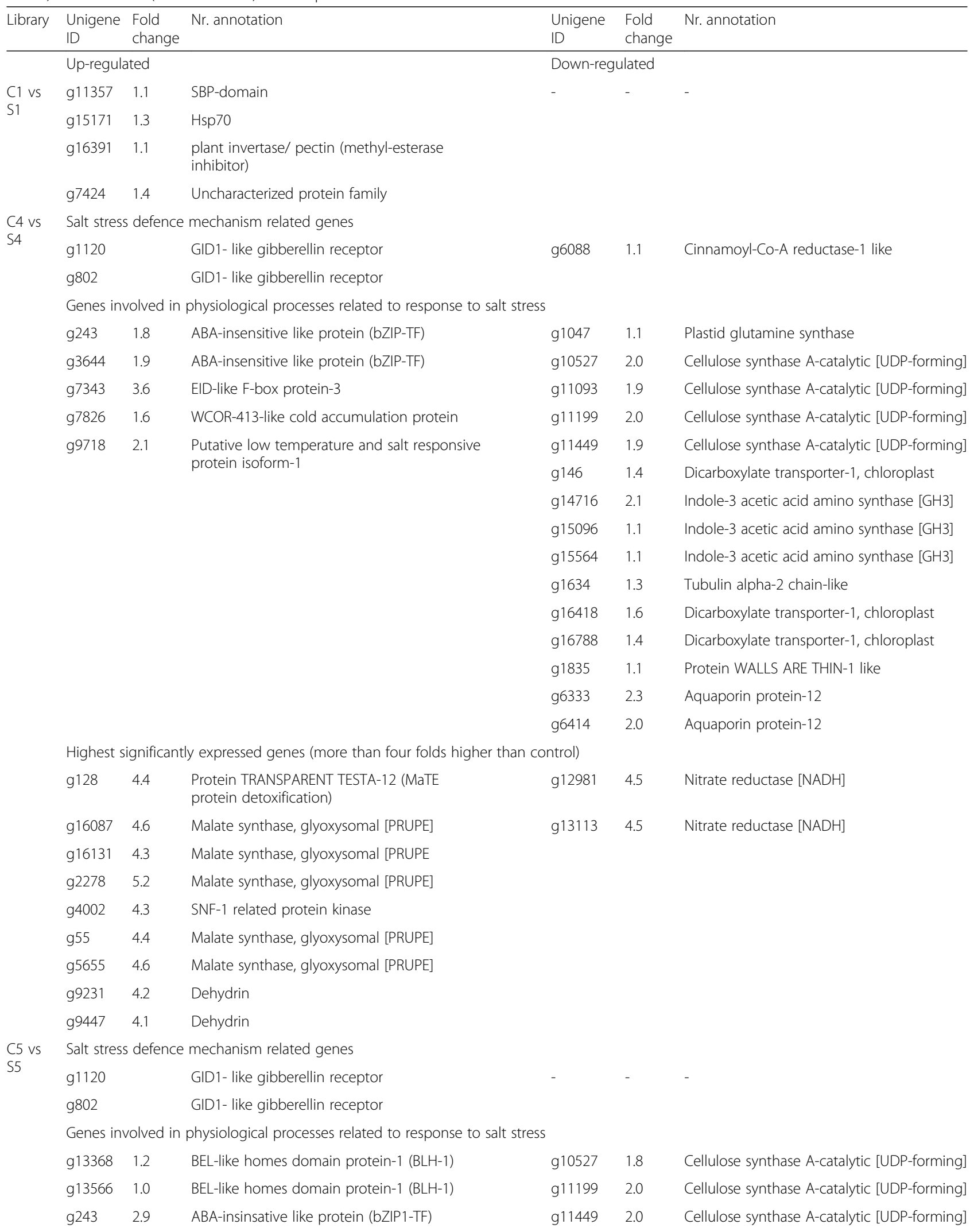


Table 4 Salt stress defence mechanism related genes, Genes involved in physiological processes related to response to salt stress and highest significantly expressed genes that were significantly up and down regulated under different durations (1, 6, 12, 48 hours) of salt stress (200 mM NaCl) as compared to control (Continued)

\begin{tabular}{|c|c|c|c|c|c|c|}
\hline Library & $\begin{array}{l}\text { Unigene } \\
\text { ID }\end{array}$ & $\begin{array}{l}\text { Fold } \\
\text { change }\end{array}$ & Nr. annotation & $\begin{array}{l}\text { Unigene } \\
\text { ID }\end{array}$ & $\begin{array}{l}\text { Fold } \\
\text { change }\end{array}$ & Nr. annotation \\
\hline & g3644 & 3.3 & ABA-insinsative like protein (bZIP1-TF) & g1634 & 1.3 & Tubulin alpha-2 chain-like \\
\hline & \multirow[t]{2}{*}{ g7343 } & \multirow[t]{2}{*}{3.9} & \multirow[t]{2}{*}{ EID-like F-box protein-3 } & $g 2681$ & 1.1 & $\begin{array}{l}\text { Enoyl-[acyl-carrier-rotein] reductase [NADH], } \\
\text { chloroplast-like }\end{array}$ \\
\hline & & & & g11093 & 2.0 & Cellulose synthase A-catalytic [UDP-forming. \\
\hline & \multicolumn{6}{|c|}{ Highest significantly expressed genes (more than four folds higher than control) } \\
\hline & g3153 & & AphC/TSA family & g11772 & 4.2 & Elongation Factor [TSF] \\
\hline & g3480 & & AphC/TSA family & g5817 & 4.5 & ACT-domain containing protein [ACR11] \\
\hline & g3682 & & Intercellular signal transduction & g8657 & 4.2 & Proline rich protein [DC2] \\
\hline & & & & g9421 & 5.5 & $\begin{array}{l}\text { Protein like isoform (plant invvertase/ pectir } \\
\text { methylesterase inhibitor) }\end{array}$ \\
\hline \multirow{28}{*}{$\begin{array}{l}\text { D6 vs } \\
\text { s6 }\end{array}$} & \multicolumn{6}{|c|}{ Salt stress defence mechanism related genes } \\
\hline & g1120 & & GID1- like gibberellin receptor & g5647 & 1.0 & Alpha/beta hydrolase (carboxylesterase) \\
\hline & g802 & & GID1- like gibberellin receptor & g5851 & 1.2 & Cinnamoyl-Co-A reductase-like \\
\hline & \multicolumn{6}{|c|}{ Genes involved in physiological processes related to response to salt stress } \\
\hline & g15315 & & Cysteine rich receptor-like protein kinase-2 & g1047 & 1.8 & Plastid glutamine synthase \\
\hline & g243 & & ABA-insensitive like protein (bZIP1-TF) & g11093 & 1.4 & Cellulose synthase A-catalytic [UDP-forming. \\
\hline & g3644 & & ABA-insensitive like protein (bZIP1-TF) & g11199 & 1.5 & Cellulose synthase A-catalytic [UDP-forming. \\
\hline & g7343 & & EID-like F-box protein-3 & g11449 & 1.5 & Cellulose synthase A-catalytic [UDP-forming. \\
\hline & \multirow{10}{*}{\multicolumn{2}{|c|}{ g9718 }} & \multirow{10}{*}{$\begin{array}{l}\text { Putative low temperature and salt responsive protein } \\
\text { isoform-1 }\end{array}$} & g146 & 1.5 & Dicarboxylate transporter-1, chloroplast \\
\hline & & & & g16196 & 1.5 & Dicarboxylate transporter-1, chloroplast \\
\hline & & & & g16418 & 1.7 & Dicarboxylate transporter-1, chloroplast \\
\hline & & & & g16788 & 1.3 & Dicarboxylate transporter-1, chloroplast \\
\hline & & & & g2795 & 1.0 & Serine-glyoxylate aminotransferase \\
\hline & & & & g3130 & 1.1 & Serine-glyoxylate aminotransferase \\
\hline & & & & g4724 & 1.1 & Uncharacterized protein sequence \\
\hline & & & & g7120 & 1.1 & Serine-glyoxylate aminotransferase \\
\hline & & & & g7168 & 1.9 & Chlorophyll a-b binding protein CP2410-A \\
\hline & & & & g7762 & 1.2 & Serine-glyoxylate aminotransferase \\
\hline & \multicolumn{6}{|c|}{ Highest significantly expressed genes (more than four folds higher than control) } \\
\hline & \multicolumn{2}{|l|}{ g10431 } & Energy production and conservation & g4007 & 5.4 & Magnisium-chelatase $[\mathrm{Ch} \mid \mathrm{H}]$, Chloroplast \\
\hline & \multicolumn{2}{|l|}{ g16087 } & Energy production and conservation & g4581 & 4.6 & Alpha expansin \\
\hline & \multicolumn{2}{|l|}{ g16288 } & Energy production and conservation & & & \\
\hline & \multicolumn{2}{|l|}{ g2278 } & Energy production and conservation & & & \\
\hline & \multicolumn{2}{|l|}{$g 2618$} & Signal transduction mechanisms & & & \\
\hline & \multicolumn{2}{|l|}{$g 2863$} & Signal transduction mechanisms & & & \\
\hline & \multicolumn{2}{|l|}{ g675 } & reverse transcription & & & \\
\hline & \multicolumn{2}{|l|}{ g7343 } & EID-like F-box protein-3 & & & \\
\hline & \multicolumn{2}{|l|}{ g9017 } & Energy production and conservation & & & \\
\hline
\end{tabular}

significantly down-regulated unigenes at $48 \mathrm{~h}$ of salt stress. Furthermore, a number of 14 unigenes were directly involved in response to salt stress included under plastid glutamine synthase-2, cellulose synthase A- catalytic
[UDP-forming], dicarboxylate transporter-1 [chloroplast], serine-glycosylate aminotransferase, chlorophyll a-b binding protein $\mathrm{CP} 2410-\mathrm{A}$ and one uncharacterized protein sequence. Out of the significantly down-regulated 
unigenes there were two unigenes which showed more pronounced effect include unigene 4007 and 4581 which have been identified as Magnesium-chelatase $[\mathrm{ChlH}]$ in chloroplast and alpha expansin protein (Table 4).

As shown in Fig. 5 there were 119 up-regulated genes present in various durations of salt stress $(6,12,48 \mathrm{~h})$. Out of these 119 unigenes, 92 genes were significantly expressed at $6,12,48 \mathrm{~h}$ of salt stress. Pfam results showed that among these 92-salt induced genes, 77 unigenes $(83.69 \%)$ were found to have a known function. A number of 5 unigenes (5.43\%) out of the significantly expressed genes showed no homology with known sequence. Furthermore, three unigenes belong to bZIP-8 transcription factor and EID-1 like F-box protein-3 were significantly expressed in leave tissues and involved in response to salt stress at $6,12,48 \mathrm{~h}$ of salt stress. On the other hand, there were 87 down-regulated unigenes in leave tissues during $6,12,48 \mathrm{~h}$ of salt stress and out of these 87 unigenes there were 42 significantly downregulated as compared to control (Fig. 6).

Based on the above mentioned results, all of these significantly unigenes during the three time points of salt stress $(6,12,48 \mathrm{~h})$ definitely have a role under salt stress conditions or may be contribute in regulating the salt response in Xuzi-8 sweetpotato cultivar.

\section{Differential expression of transcription factors (TFs) regulated by salt stress}

There were 4, 1202, 764 and 2195 transcription factors differentially regulated DEGs by salt stress at different time points including $1,6,12,48 \mathrm{~h}$ of salt stress. During the first hour of salt stress there were 4 different TFs up-regulated in the salt treated plants which were included under SBP, LIM, NAC and FAR1 TFs families.

At $6 \mathrm{~h}$ of salt stress the number of TFs increased giving 1202 TFs including 529 up-regulated and 672 down-regulated TFs. The most pronounced TFs regulated after $6 \mathrm{~h}$ of salt stress (expressed 5 folds than control) was aligned to malate synthase which is responsible for energy production and conversion. Followed by 9 unigenes expressed with 4 folds higher than control which were included under mTERF and WRKY TFs families. Furthermore, 38 unigenes belongs to NF-Y-> NF-YC, C2C2- > C2C2-GATA, DBP, C3H, WRKY, Tify, SNF2 and HB-> HB-HD-ZIP TFs families were upregulated with 3 folds than control. On the other hand 672 TFs down regulated in leaf tissues of treated plants lower than control. The lowest down regulated unigenes were belonged to $\mathrm{C} 3 \mathrm{H}$ and SET TFs families (namely: Nitrate reductase $[\mathrm{NADH}]$ and Auxin-binding protein) which are involved in oxidation-reduction process and auxin-activated signalling pathway, respectively. Unigenes belong to GNAT TF family including Aquaporin PIP2-7 and Chlorophyll a-b binding protein 48 were down regulated as compared to control. GNAT TF family are involved in Carbohydrate transport and metabolism, water transport and water channel activity. MADS-> MADS-M-type TF family aligned unigenes (down-regulated with 3 folds lower the control) are involved in lipid transport and metabolism.

After $12 \mathrm{~h}$ of salt stress, a number of $673 \mathrm{TFs}$ were differentially regulated in treated plants than control plants including 341 up-regulated and 422 down-regulated TFs. The up-regulated TFs were aligned to different TF families including HB- > HB-HD-ZIP (13 unigenes), C2C2$>$ C2C2-GATA (12 unigenes), DBP (11 unigenes), SNF2 (10 unigenes), bZIP and $\mathrm{C} 2 \mathrm{H} 2$ (8 unigenes), NF-Y-> NF-YC (6 unigenes) and WRKY (5unigenes). The highest up-regulated TFs (4 folds higher than control) was aligned to C2C2- > C2C2-GATA family namely nucleoredoxin 2 including Thioredoxin-like domain which is involved in intracellular signal transduction. Followed by DBP, bZIP and SNF2 TFs families including genes nucleoredoxin 2, protein phosphatase 2C, ABSCISIC ACID-INSENSITIVE 5-like protein 5 (bZIP8) and EID1like F-box protein, respectively. On the other hand, there were 422 unigenes down regulated were classified as TFs including AP2/ERF- > AP2/ERF-ERF (31 unigenes), MYB$>$ MYB-related (22 unigenes), GNAT (15unigenes), C3H (13 unigenes), LIM (11 unigenes), Trihelix (11unigenes) and SWI/SNF-BAF60b (10 unigenes). MYB- > MYB-related (ACT domain-containing protein ACR11) and Trihelix (14 kDa proline-rich protein DC2.15-like) TFs families were the lowest expressed among all. In addition there were a number of 14 unigenes significantly downregulated 3 folds less than control which were aligned to geraniol 10-hydroxylase-like protein, Elongation factor Ts, cytochrome P450, Expansin-A1, photosystem II protein D2, abscisic acid receptor PYL4-like, $14 \mathrm{kDa}$ proline-rich protein DC2.15-like and gibberellin induced protein. These genes were belonged to HB- > HB-other, bHLH, LIM, MYB, Trihelix and mTERF TF families.

The number of TFs induced by exposure to $48 \mathrm{~h}$ of salt stress was the highest among all time points (2194 TFs) including 663 up-regulated and 1531 downregulated unigenes. The up-regulated TF families were C3H (26 unigenes), WRKY (16 unigenes), bZIP (15unigenes), NAC (15 unigenes), FAR1 (15 unigenes), HB-> HB-HD-ZIP (13 unigenes), PHD (13 unigenes), C2H2 (12 unigenes), SNF2 (11 unigenes), AP2/ERF- > AP2/ ERF-ERF (11 unigenes) and B3-> B3 (11 unigenes). On the contrary, there were 1531 down regulated TFs included under different TFs families. One unigene encoded magnesium-chelatase subunit $\mathrm{ChlH}$ (chloroplastic) was expressed five folds lower than control was belonging to Jumonji TF family which is involved in chlorophyll biosynthetic process and work as a coenzyme transport and metabolism. A unigene aligned to 
Expansin-A2 belongs to LIM TFs family was downregulated 4 folds lower than control which found to be plant-type cell wall organization. In addition, there were 33 unigene expressed with 3 folds lower than control.

\section{Protein kinases (PKs) differentially expressed under salt stress}

In sweetpotato at the time of salt stress, PKs include many genes and play a vital role in phosphorylation process and act as a signal transductor/receptor proteins in membranes.

SNF1-related protein kinase was up-regulated during $6 \mathrm{~h}$ (7 unigenes) and $48 \mathrm{~h}$ (4 unigenes) of salt stress expressed in two forms including SNF1-related protein kinase catalytic subunit alpha KIN10-like and SNF1-related protein kinase regulatory subunit gamma-1. One unigene aligned to Phosphatidylinositol 4-phosphate 5-kinase was upregulated during 6 and $48 \mathrm{~h}$ after salt stress. A number of 19 unigenes were aligned to cyclin-dependent kinase which were up-regulated at 6 and $48 \mathrm{~h}$ of salt stress. CBLinteracting protein kinase 10 aligned genes were downregulated. Cysteine-rich repeat secretory protein 3 is a salt stress response gene was up-regulated starting from $6 \mathrm{~h}$ of salt stress. G-type lectin S-receptor-like serine/threonineprotein kinase aligned genes (14 unigene) were upregulated only at $12 \mathrm{~h}$ of salt stress. A number of 6 unigenes aligned to wall-associated receptor kinase were downregulated starting from 6 till $48 \mathrm{~h}$ of salt stress. A number of 46 unigenes were classified under LRR receptor-like protein kinase were up-regulated at 12 and $48 \mathrm{~h}$ of salt stress.

\section{Photosynthesis related genes under salt stress}

Leaf is the factory of the plant that provide the plant with necessary energy for different physiological processes through photosynthesis. Genes related to photosynthetic parameters including gas exchange, pigments are affected seriously by salt stress. The current transcriptome sequencing results showed that there were 441 unigenes identified to be involved in photosynthetic related processes or pathways. Exposing he plant for $200 \mathrm{mM}$ of Nacl for $1 \mathrm{~h}$ didn't significantly affect photosynthesis process or its related genes. Most of genes involved in photosynthesis process were down regulated including 88, 20 and 239 DEGs at 6, $12,48 \mathrm{~h}$ of salt stress as compared to control. While, a very low number of DEGs were up-regulated including 8 and 5 DEGs at 6 and $48 \mathrm{~h}$ of salt stress as compared to control. At $6 \mathrm{~h}$ of salt stress a number of 8 unigene aligned to Fructose-1,6-bisphosphatase (cytosolic) were up-regulated. Furthermore, after $48 \mathrm{~h}$ there were only 5 up-regulated DEGs which were aligned to Aconitate hydratase (cytoplasmic) and heme oxygenase 1 (chloroplastic). In addition there was one gene was up-regulated during 6 and $48 \mathrm{~h}$ of salt stress which is encoded to stress enhanced protein 2 (chloroplastic). On the other hand, there were 10 unigenes down regulated at $6,12,48 \mathrm{~h}$ which were aligned to ADPglucose pyrophosphorylase beta, thylakoid lumenal $29 \mathrm{kDa}$ protein (chloroplastic), chlorophyll a-b binding protein (chloroplastic) and rhodanese-like domain-containing protein 9 (chloroplastic). At $6 \mathrm{~h}$ the most pronounced downregulated gene as compared to control was chlorophyll $a-b$ binding protein which is common in all time points beside Protochlorophyllide reductase (chloroplastic). At $12 \mathrm{~h}$ beside to the common genes, serine hydroxymethyltransferase (mitochondrial), mitogen-activated protein kinase, photosystem II protein D2, Photosystem I reaction center subunit $\mathrm{XI}$ (chloroplastic) and $\mathrm{NAD}(\mathrm{P}) \mathrm{H}$-quinone oxidoreductase (chloroplastic) were down regulated. At $48 \mathrm{~h}$ the lowest down-regulated gene was magnesium-chelatase subunit ChlH (chloroplastic) followed by chlorophyll a-b binding protein and photosystem I reaction center subunit XI (chloroplastic).

\section{SSR and SNP identification}

For further application of sweetpotato SSRs and SNPs were discovered using assembled transcriptomes (Fig. 7a and b). A total of 24,559 SSRs were identified in transcriptomes present in 15,976 sequences. Furthermore, the numbers of sequence containing more than one SSR were 5762 and the numbers of SSRs present in compound formation were 2215. In addition, the major types of the identified SSRs were mono-nucleotide (19,380), dinucleotide (2791), tri-nucleotide (2199), tetra-nucleotide (123), penta-nucleotide (33) and hexa-nucleotide (25). The most SSR motif was A/T (19282) followed by AG/CT (1964), AT/AT (669), AAG/CTT (596), CCG/CGG (330), AAT/ATT (309), ATC/ATG (247) and AGC/CTG (217). A total of 562,174 SNPs between transcriptomes were identified, among which 348,495 were transitions, and 213,679 were transversions. These SSRs and SNPs identified in this study provided a valuable resource for future studies on genetic linkage mapping and the analysis of interesting traits in sweetpotato.

\section{Experimental validation by qRT-PCR}

To confirm the reliability of the illumine sequencing, six unigenes were randomly selected for quantitative RTPCR assays including unigene299, 2083, 2359, 3153, 6075 and 6453. The results showed that the DEG results and expression levels have the same tendency among all unigenes (Fig. 8). The unigenes 299, 2359, 3153 and 6075 were up-regulated in both qPCR results and DEG analysis giving the lowest values at the first time point ( $1 \mathrm{~h}$ of salt stress) then started to increase till $48 \mathrm{~h}$ of salt stress. On the other hand, the unigenes 2083 and 6453 were down regulated with an expression level one fold lower than control samples which was in agree with the DEG results (Fig. 8a-f). 


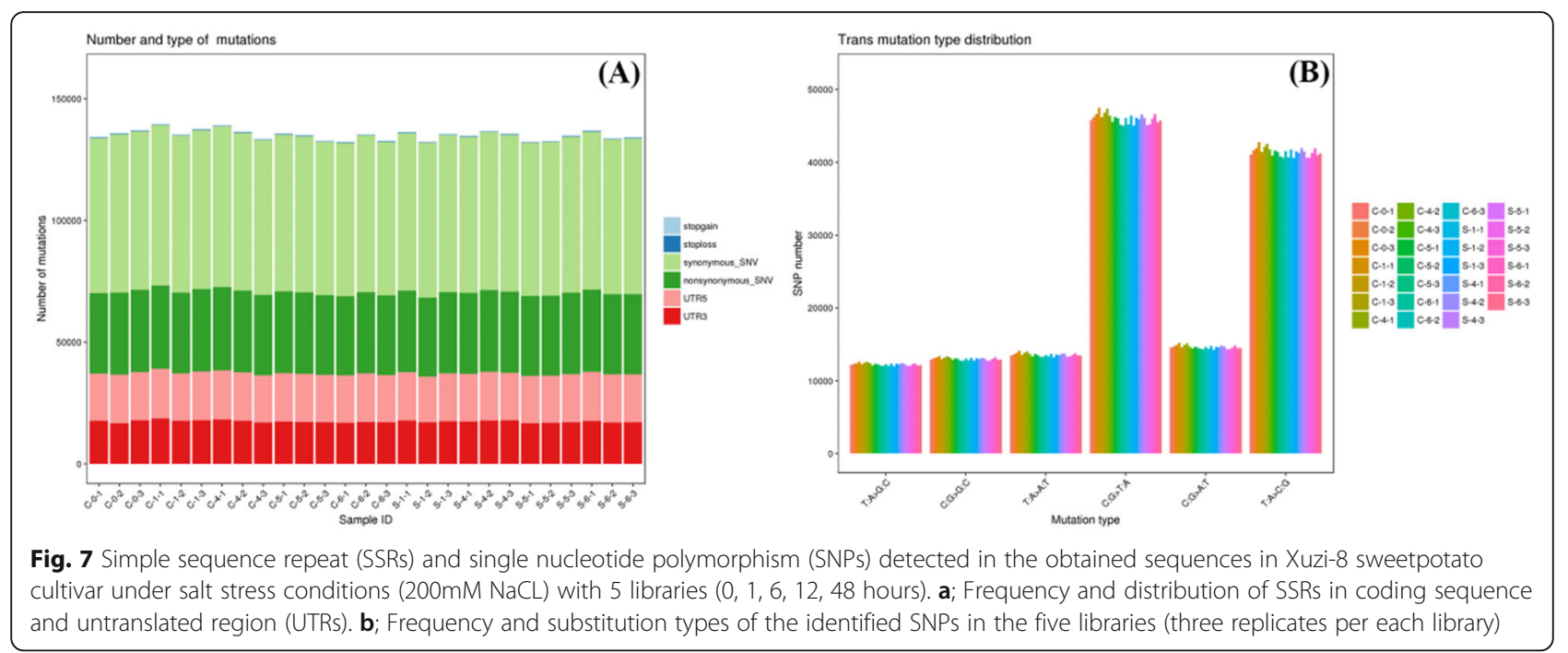

\section{Discussion}

Sweetpotato is a hexaploid heterozygous non-model crop with a complex genome lacking high quality reference genome [13]. To date the current understanding of the complex physiological and molecular mechanisms of salt tolerance in sweetpotato remains limited [14, 15]. High throughput RNA sequencing is required for identification of candidate genes involved in salt stress tolerance. In addition, it will be helpful for better understanding of salts stress tolerance mechanisms in sweetpotato [5].

In the present study, deep sequencing analyses of PFSP under different durations of salt stress were characterized as compared to control. Using second and third generation technology, Illumina sequencing generated $170,344,392$ clean high-quality long reads that were assembled into 15,998 unigenes with an average length 2178 base pair which considered relatively longer than only NGS studies which ranged between 1000 and 1300 base pairs as an average length for the obtained sequence [16-19]. A percent of $96.55 \%$ of the obtained unigenes were functionally annotated in the NR protein database, while there were 537 unigenes failed to hit any homologs which may be considered as new protein sequence which had not been characterized previously in sweetpotato.

The current results revealed that there are similarity with the available sweetpotato genome sequence at a

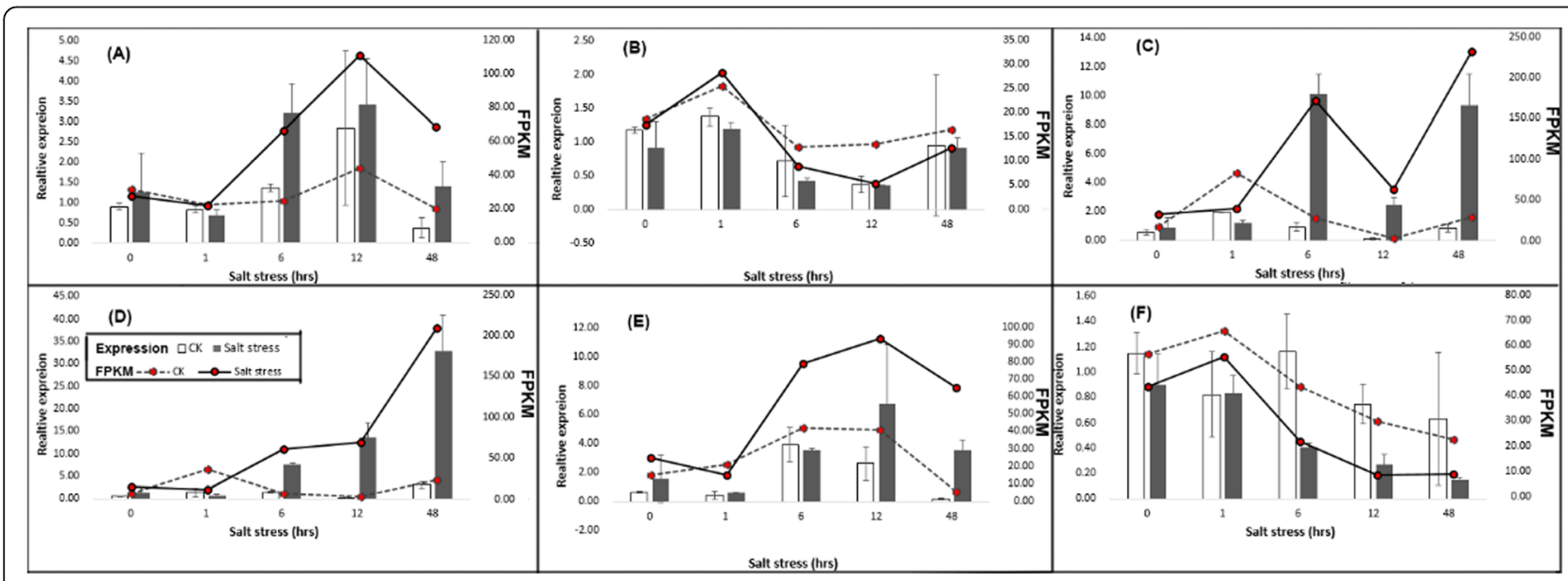

Fig. 8 qRT-PCR validation of the salt induced fold changes detected in Xuzi-8 sweetpotato cultivar using RNA-seq. Standard error bars are showed for the expression values determined by qRT-PCR. The expression levels determined by qRT-PCR and DEG values of the genes (protein disulfide isomerase, Actin-12 (ACT12), nucleoredoxin 2 isoform X1, Thioredoxin-like domain, CTD small phosphatase-like protein 2 and Redoxinwere presented) at (a), (b), (c), (d), (e) and (f), respectively. Expression values determined by qRT-PCR are presented as columns and DEGs are presented as curves 
percent of $3.07 \%$. That's mean that $96.93 \%$ of our obtained sequence are not present on the sweetpotato available online data. Therefore, the current results will contribute to improve sweetpotato genome annotation and facilitate the discovery of genetic resources that are responsible for salt stress in hexaploid sweetpotato.

The results in Fig. 3 showed that the most dominant Go-terms which were identified during the different durations of salt stress included metabolic, cellular, single organism and response to stimulants. These results are in agree with [20] in diploid halophytic sweetpotato, [21] in Ipomoea imperati [22]. Furthermore, COG results indicated that the highest number of genes was categorized as "post-translational modification, protein turnover, chaperones" in our study. Which mean that, sweetpotato plants under salt stress conditions, starts the key mechanisms of chemical modifications including regulating of enzymes activity, localization and interaction with other cellular molecules such as proteins, nucleic acid, lipids and co-factors [23]. Furthermore, protein turnover related genes control the balance between protein biosynthesis and degradation, which is very important for determining the resistance or sensitivity of the plant to salt stress. That's may be due to if the protein biosynthesis is more than breakdown indicates an anabolic state, in this case the plant tolerance to stress will be enriched [24-27]. Moreover, Protein chaperones' major function is to prevent or correct damage caused by miss-folding due to salt stress [28-31]. The second COG group of genes involved in "signal transduction mechanisms" which is mainly transforming the certain stimulus induced by salt stress into a biochemical signal which activates more genes specifically involved in salt stress tolerance [32].

\section{The regulatory mechanisms and salt resistance related genes in sweetpotato}

From the molecular aspects, salt tolerance in sweetpotato starts when the plant exposed to salt stress, the action taken including activating the transcriptional control inside the nucleus of the cells, sensing and signaling, cellular influx and detoxification mechanisms activated [33].

TFs play an essential role in regulating many different signal transduction pathways in plants under stress by activating the expression of specific genes [34]. In the current study, the response of leaf tissues was lower during the first hours of salt stress and there were only four TFs significantly up-regulated with one fold higher than control. These four unigenes including SBP TFs have important role in leaf development, vegetative phase change and may be have a role in stress response [35]. The second unigene was aligned to HSP70 which is essential regulator to maintain internal cell stability and prevent aggregation under physical or chemical pressure [36]. The plant invertase/ pectin methyl-esterase inhibitor affects growth and development consisting with its activities such as stress response. In addition, pectin methyl-esterase determines the solidity of cell wall including root development and permeability [37]. These four genes considered as early response genes for stress in leaf tissues [38] which can improve stress tolerance at the first phase of stress. This indicate that Xuzi-8 sweetpotato as a tolerant cultivar slightly sensed osmotic stress at the first hour of salt stress. However, only four TFs were up-regulated which considered necessary in linking salt sensory pathways to salt tolerance as well as other types of stress.

In addition the current results showed that a core sets of TF family genes were differentially expressed in leaf tissues during 6, 12 and $48 \mathrm{~h}$ of salt stress including C3H, WRKY, bZIP, NAC, FAR1, HD-ZIP, PHD, C2H2, SNF2, AP2/ERF, MYB and B3. In agree with our results Geng Y. et al., [39] reported that most of stress induced transcriptional changes occurs at least after $3 \mathrm{~h}$ of salt stress exposure. These TFs in turn are regulating the expression levels of different genes that may ultimately enrich salt tolerance in sweetpotato [39].

In the current study, at $6 \mathrm{~h}$ of salt stress the most pronounced TFs were malate synthase, mTERF and WRKY. These three TFs have an important role in plant physiology under salt stress conditions [40]. Followed by SNF-1 related protein kinase (represent an interface between metabolic and stress signaling) [41] and dehydrin (play a major role in recovery of drought and salt stress) [42]. In addition to TFs, at $6 \mathrm{~h}$, there were protein TRANSPARENT TESTA-12 which control flavonoid sequestration [43] and Protein DETOXIFICATION is delay the development of disorders associated with stress [43]. Furthermore, ABA insensitive-like protein is acting as a positive component in glucose signal transduction [43] and EID-like F-box protein family is a known drought and salt response regulation after $6 \mathrm{~h}$. WCOR 413-like protein is responsible for protection of plasma membrane against dehydration which has been discovered earlier in sweetpotato genome, these findings are in agree with [43] in wheat. The putative salt stress responsive protein isoform- 1 , which play a critical role in salt stress tolerance after $6 \mathrm{~h}$, has been discovered before in sweetpotato genome [44]. Thus, we can conclude that at $6 \mathrm{~h}$ the plant start to activate the TFs which regulate genes involved in salt stress tolerance, protein kinases that save the metabolic activity and activate stress signaling beside protein detoxifications which delay the harmful effect of salt stress and reduce the excess absorption of $\mathrm{NaCL}$.

The highest regulated TFs at $12 \mathrm{~h}$ of salt stress as compared to control was nucleoredoxin 2 including 
Thioredoxin-like domain (AhpC/TSA TFs) which is responsible for signal transduction which in role constitutes an enzymatic defense against salt stress. In addition, at $12 \mathrm{~h}$ of salt stress, BEL-1 like homes domain protein-1 (BLH-1) contribute in regulating a range of developmental processes under salt stress, bZIP-8 transcription factor is a key components in response to a wide range of abiotic stresses, including high salinity through regulating $\mathrm{ABA}$ concentration. In addition $\mathrm{ABA}$ is responsible for preventing the lateral root elongation into surrounding media with high salt concentration. Furthermore, EID-1 like F-box protein-3 were expressed with high level as compared to control [43]. Therefore, at $12 \mathrm{~h}$ the genes involved in sensing and signaling were more expressed, beside the TFs which are involved in hormonal regulation which have a major role in defense mechanisms against salt stress.

In the current study, as compared to control, among the other time points, the number and expression level of TFs at $48 \mathrm{~h}$ was the highest. These TFs families which have a strong relation with salt stress tolerance at $48 \mathrm{~h}$ including C3H, WRKY, bZIP, NAC, FAR1, HB- > HBHD-ZIP, PHD, C2H, SNF2, AP2/ERF- > AP2/ERF-ERF, EID-1 like F-box protein-3 and B3- > B3. In addition, the unigenes which responded to salt stress were Cysteinrich receptor-like protein kinase-2, low temperature and salt responsive protein isoform. Furthermore, the highest expressed genes in leave tissues were belong to importin-5 malate synthase, glyoxysomal, protein phosphatase 2C-37 like, O-acyltransferase WSD-1-like, and NADH-dehydrogenase [45-48].

\section{Photosynthesis related genes}

Photosynthesis is the most significant physiological process for the plant life and during all growth stages is affected by stress factors [33]. In the current results, it was interesting to note that most of DEGs related to photosynthesis were down-regulated, while, a very low number of unigenes were up-regulated. The stress enhanced protein 2 (chloroplastic) was significantly up-regulated at 6 and $48 \mathrm{~h}$ of salt stress. In addition, fructose-1,6-bisphosphatase (cytosolic) was up-regulated after $6 \mathrm{~h}$ of salt stress which is involved in carbohydrate transport and metabolism and Aconitate hydratase (cytoplasmic) and Heme oxygenase 1 (chloroplastic) were up-regulated at $48 \mathrm{~h}$ of salt stress. According to our results, Heme oxygenase 1 (chloroplastic) was up-regulated which is responsible for inorganic ion transport and metabolism that's in role counteract the reduction of water potential resulting from osmotic components of enhanced salinity [39]. On the other hand, the number of genes related to photosynthesis was 88 at $6 \mathrm{~h}$ off salt stress decreasing into only 20 unigenes at $12 \mathrm{~h}$ and reached to the maximum at $48 \mathrm{~h}$ of salt stress. There were 10 unigenes common in the three time points $6,12,48 \mathrm{~h}$ including ADP-glucose pyrophosphorylase beta, thylakoid lumenal $29 \mathrm{kDa}$ protein (chloroplastic), chlorophyll a-b binding protein (chloroplastic) and rhodanese-like domain-containing protein 9 (chloroplastic). Furthermore, the down-regulated unigenes were involved in amino acid transport and metabolism, carbohydrate transport and metabolism, cell wall/membrane/envelope biogenesis, secondary metabolites biosynthesis, transport and catabolism, energy production and conversion, inorganic ion transport and metabolism and coenzyme transport and metabolism. To some extent, genes involved in the above mention physiological processes are mediated by dynamic changes in photosynthesis [39]. Therefore, according to hour results and in agree with previous researches, photosynthesis process severely affected by salt stress starting from $6 \mathrm{~h}$ and the effect increase with increasing the stress duration.

Taken together, TFs (bHLH, bZIP, C2H2, C3H, C3H4, ERF, MYB, NAC, TSA and WRKY) regulates the differentially expressed genes that related to salt stress tolerance and PKs act as a signal transductor/receptor proteins in membranes. Furthermore, protein detoxifications have damage control and repair associated with stress. In addition genes related to hormonal balance have a major role in determining the level of plant tolerance. According to our results, due to the high expression of these genes including TFs, PKs, Protein Detox and hormones related genes enriched the salt tolerance in Xuzi-8 sweetpotato cultivar.

\section{Conclusions}

In the present study, de novo was constructed and characterized the transcriptomes of sweetpotato challenged with salt stress and identified 15,976 unigenes, generating a broad survey of genes involved in salt stress resistance. DEG profiling analysis at the significance level identified number of 4, 479, 281, 508 up-regulated and $0,567,301,1335$ down-regulated unigenes. Functional analysis of the obtained sequence showed that the main TFs families involved salt stress tolerance including bHLH, bZIP, C2H2, C3H, C3H4, ERF, MYB, NAC, TSA and WRKY. PKs act as a signal transductor/receptor proteins in membranes and protein detoxifications have damage control and repair associated with stress. The current transcriptome sequencing data of hexaploid sweetpotato under salt stress conditions can provide a valuable resource for sweetpotato breeding research and focus on novel insights into sweetpotato responses to salt stress. In addition, it offers new candidate genes or markers that can be used as a guide to the future studies attempting to breed salt tolerance sweetpotato cultivars. 


\section{Methods}

\section{Plant materials}

Xuzi-8, a high quality, early mature cultivar with green leaves and purple flesh storage roots, and salt tolerance, was used in this experiment. Its storage roots contain $6 \%$ soluble sugar and more than $80 \mathrm{mg}$ anthocyanin $/ 100 \mathrm{~g}$ fresh weight. It have a strong vegetative growth and its leaves contain 398.31, 130.75 and $39.27 \mathrm{mg} / 100 \mathrm{~g}$ dry weight of chlorophyll a, chlorophyll b and carotenoids, respectively [49]. This cultivar was obtained from Xuzhou Institute of Agricultural Sciences in Jiangsu Xuhuai District, China.

\section{Treatments and experimental design}

Apical stem cuttings $(15-20 \mathrm{~cm})$ of Xuzi- 8 cultivar taken from 2 months age healthy sweetpotato plants growing in the field. Stem cuttings were let to grow in hydroponics culture using Hoagland nutrient medium till having $1 \mathrm{~cm}$ pencil roots (about 3 weeks). Seedlings were exposed to salt stress $(200 \mathrm{mM} \mathrm{NaCl}$ were added to Hoagland solution) for $0,1,6,12,48 \mathrm{~h}$ which were chosen according to our previous study [50]. $0.1 \mathrm{~g}$ sample (three replicates per each sample) was taken from the upper third leaf after $0,1,6,12$, $48 \mathrm{~h}$ from salt treated and untreated plants for the next generation sequencing. For the third-generation sequencing, samples was collected from the upper third leaf at the same time points (three replicates for each sample). All collected samples were immediately frozen in liquid nitrogen and stored at $-80{ }^{\circ} \mathrm{C}$ until processing for RNA extraction and all treatments were done in triplicate.

\section{RNA extraction}

Total RNA was extracted from collected samples with the TRIZOL method (Life technologies, Carlsbad, CA) according to the manufacturer's protocol. RNA degradation and contamination were monitored on $1 \%$ agarose gels. RNA purity was checked using the Nanophotometer $^{\oplus}$ spectrophotometer (IMPLEN, CA, USA). RNA concentration was measured using Qubit ${ }^{\oplus}$ RNA Assay Kit in Qubit $^{\oplus}$ 2.0 Fluorometer (Life Technologies, CA, USA). RNA integrity was assessed using the RNA Nano 6000 Assay Kit of the Agilent Bioanalyzer 2100 system (Agilent Technologies, CA, USA).

\section{Library preparation for Transcriptome analysis}

For second generation sequencing, a total amount of $3 \mu \mathrm{g}$ RNA per sample was used as input material for the RNA sample preparations. Sequencing libraries were generated using NEBNext $^{\circ}$ Ultra $^{\text {TM }}$ RNA Library Prep Kit for Illumina ${ }^{\circ}$ (NEB, USA) following manufacturer's protocol and index codes were added to attribute sequences to each sample. For third generation sequencing RNA was extracted from different time points and equal amount of each sample were mixed to produce one library beside to the control library. Briefly, mRNA was purified from total RNA using poly-T oligo-attached magnetic beads. Fragmentation was carried out using divalent cations under elevated temperature in NEBNext First Strand Synthesis Reaction Buffer(5X). First strand cDNA was synthesized using random hexamer primer and M-MuLV Reverse Transcriptase (RNase H-). Second strand cDNA synthesis was subsequently performed using DNA Polymerase I and RNase $H$. Remaining overhangs were converted into blunt ends via exonuclease/polymerase activities. After adenylation of 3' ends of DNA fragments, NEBNext Adaptor with hairpin loop structure were ligated to prepare for hybridization. In order to select cDNA fragments of preferentially $150 \sim 200 \mathrm{bp}$ in length, the library fragments were purified with AMPure XP system (Beckman Coulter, Beverly, USA). Then $3 \mu$ l USER Enzyme (NEB, USA) was used with size-selected, adaptor-ligated cDNA at $37^{\circ} \mathrm{C}$ for 15 min followed by $5 \mathrm{~min}$ at $95^{\circ} \mathrm{C}$ before PCR. Then PCR was performed with Phusion High-Fidelity DNA polymerase, Universal PCR primers and Index (X) Primer. At last, PCR products were purified (AMPure $\mathrm{XP}$ system) and library quality was assessed on the Agilent Bioanalyzer 2100 system.

\section{Clustering and sequencing}

The clustering of the index-coded samples was performed on a cBot Cluster Generation System using TruSeq PE Cluster Kit v3-cBot-HS (Illumia) according to the manufacturer's instructions. After cluster generation, the library preparations were sequenced on an Illumina platform and paired-end reads were generated.

\section{Data analysis quality control}

Raw data (raw reads) of fastq format were firstly processed through in-house perl scripts. In this step, clean data (clean reads) were obtained by removing reads containing adapter, reads containing ploy- $\mathrm{N}$ and low-quality reads from raw data. At the same time, Q20, Q30, GCcontent and sequence duplication level of the clean data were calculated. All the downstream analyses were based on clean data with high quality.

\section{Transcriptome assembly}

Transcriptome sequencing was accomplished based on both NGS and 3rd GS, and TPM, FPKM, RPKM and fold change (FC) were recorded for each replicate of each library separately. Obtained sequence from NGS and 3rd GS was aligned and similar sequence data from all libraries/samples were pooled [51] and ultimately 
used for further analysis using Trinity [52] with min kmer_cov set to 2 by default and all other parameters set default.

\section{Gene functional annotation}

Gene function was annotated based on the following databases: $\mathrm{Nr}$ (NCBI non-redundant protein sequences, https://www.ncbi.nlm.nih.gov/) with E-value cut-off of le5; Nt (NCBI non-redundant nucleotide sequences https:// www.ncbi.nlm.nih.gov/) with E-value cut-off of le-5 ; Pfam (Protein family, http://pfam.sanger.ac.uk/) with E-value cut-off of le-2; KOG/COG (Clusters of Orthologous Groups of proteins, https://www.ncbi.nlm.nih.gov/cog/) with E-value cut-off of le-3 and Swiss-Prot (A manually annotated and reviewed protein sequence database, http:// www.ebi.ac.uk/uniprot/) with E-value cut-off of le-5.

Based on the NR and Pfam annotations, Blast2GO (v2.5) was used to obtain GO (Gene Ontology) annotations (http://www.geneontlology.org) according to the molecular functions, biological processes and cellular component ontologies [53]. GO enrichment analysis of the differentially expressed genes (DEGs) was implemented by the GO-seq $\mathrm{R}$ packages based Wallenius non-central hyper-geometric distribution [54], which can adjust for gene length bias in DEGs. KOBAS software (http://www.genome.jp/kegg/) were used to test the statistical enrichment of DEGs in KEGG [55].9. SNP calling and SSR detection.

Picard - tools v1.41 and samtools v0.1.18 were used to sort, remove duplicated reads and merge the bam alignment results of each sample. GATK software was used to perform SNP calling. Variants were kept for quality using the following parameters (1) mapping quality filter equal to PASS; (2) Quality Depth (QD) > 2; (3) Mapping Quality (MQ) > 40; (5) QUAL > 30; Moreover, variants were further filtered if coverage $<10$, if cluster SNPs more than 2 in 5 bp window, if SNP around Indel within $5 \mathrm{bp}$.

SSR of the transcriptome were identified using MISA (http://pgrc.ipk-gatersleben.de/misa/misa.html), and primer for each SSR was designed using Primer3 (http://primer3.sourceforge.net/releases.php).

\section{Quantification of gene expression levels and differential expression analysis}

Gene expression levels were estimated by RSEM [56] for each sample. Clean data were mapped back onto the assembled transcriptome. Read count for each gene was obtained from the mapping results.

Differential expression analysis of two conditions/ groups was performed using the DESeq2R package DESeq2 rovide statistical routines for determining differential expression in digital gene expression data using a model based on the negative binomial distribution. The resulting $P$ values were adjusted using the Benjamini and Hochberg's approach for controlling the false discovery rate. Genes with an adjusted $P$-value $\leq 0.001$ found by DESeq2 were assigned as differentially expressed [57]. Q-value $\leq 0.001 \& \mid \log 2 \mathrm{FC}$ (foldchange) $\mid \geq$ 1 was set as the threshold for significantly differential expression.

\section{Quantitative and real time (qRT-PCR) validation}

To confirm the reliability of the illumine sequencing, six unigenes were randomly selected for quantitative RTPCR assays. Total RNA was extracted from collected samples with the TRIZOL method (Life technologies, Carlsbad, CA) and reverse transcription were done according to the manufacturer's protocol. RT-qPCR was performed using the method described by [58] and Ubi3 (AY486137.1) was used as internal control (reference gene) [59]. qPCR primers of each unigene were designed

Table 5 RT-qPCR primers for validation experiment

\begin{tabular}{llll}
\hline Unigene code & Direction & Sequence $\left(5^{\prime}\right.$->3') & Length \\
\hline g299 & Forward & TाTCGTAATCCTGGCGGCG & 20 \\
& Reverse & ACCGTGCGGAGAAGTGGT & 20 \\
g2083 & Forward & CCGCCCGAGAGGAAGTACAG & 20 \\
g2359 & Feverse & GGGCCAGACTCGTCGTACTC & 20 \\
& Forward & GGAGGCGTTGCTGCTTACC & 20 \\
g3153 & Reverse & TGCCCTCGACGTGAACCTT & 20 \\
& Forward & TTCGACGCCTACTTCGGAC & 20 \\
g6075 & Reverse & GTCCGGCCCGAGAATACCA & 20 \\
& Forward & TCTAGCAATGGTGCTGCGGA & 20 \\
& Reverse & AGCCCTACTGCTCCAACTACG & 20 \\
\hline
\end{tabular}


using primer 5 software are shown in Table 5 . All reactions were prepared in triplicates and relative expression was calculated using $2^{\wedge}(-\Delta \Delta C \mathrm{t})$ method with the expression normalised against the internal reference gene (Tubulin2) [60].

\section{Supplementary information}

Supplementary information accompanies this paper at https://doi.org/10. 1186/s12864-020-6524-1.

Additional file 1. GO function annotations including number of unigenes and unigenes ID; COG classes and functions with unigenes ID.

Additional file 2. Detailed GO function classification of differentially expressed unigenes between control and different treatments.

Additional file 3. The Most enriched GO terms during the different time points as compared to the control.

Additional file 4. KEGG different pathways including unigenes belonging for different pathways

\section{Abbreviations}

3rd GS: Third generation sequencing; FDR: False discovery rate; FPKM: Fragments per kilo base million; GO: Gene ontology; KOG/ COG: Clusters of orthologous groups of proteins; NGS: Next generation sequencing; Nr: NCBI non-redundant protein sequences; Nt: NCBI nonredundant nucleotide sequences; PFSP: Purple flesh sweetpotato; RPKM: Reads per kilo base million; TPM: Transcripts per kilo base million

\section{Acknowledgements}

Not applicable.

\section{Authors' contributions}

$L Q$, and MHA designed the study. MHA conducted the experiments, analyzed the results, performed the figures and wrote the manuscript. LQ, $L Y, H A$ and $M Q$ revised the manuscript. TW, KM, YH, GF, WX and ZY assisted in the experimental procedures. All authors have read and approved the manuscript.

\section{Authors' information}

Not applicable

\section{Funding}

This work was supported by the earmarked fund for China Agriculture Research System (CARS-10, Sweetpotato), the "333 talent project" fund of Jiangsu Province (BRA2016265), China, Talented Young Scientist Program of Ministry of Science and Technology and the Natural Science Foundation of Jiangsu Province (Grants No BK20161162). The funding bodies played no role in the design of the study and collection, analysis, and interpretation of data and in writing the manuscript.

\section{Availability of data and materials}

Not applicable.

\section{Ethics approval and consent to participate}

Not applicable.

\section{Consent for publication}

Not applicable.

\section{Competing interests}

The authors declare that they have no competing interests.

\section{Author details}

${ }^{1}$ Xuzhou Institute of Agricultural Sciences in Jiangsu Xuhuai District / Key Laboratory of Biology and Genetic Improvement of Sweetpotato, Ministry of Agriculture / Sweetpotato Research Institute, CAAS, Xuzhou 221131, Jiangsu, China. ${ }^{2}$ Department of Horticulture, Faculty of Agriculture, Zagazig University, Zagazig, Sharkia 44511, Egypt. ${ }^{3}$ Plant pathology department, Agriculture and
Biology research division, National research center, Giza, Egypt. ${ }^{4}$ Department of Plant Breeding and Genetics, Bahauddin Zakariya University, Multan 60000, Pakistan.

Received: 18 October 2019 Accepted: 21 January 2020

Published online: 04 March 2020

\section{References}

1. Troung VD, Avula RY, Pecota K, Yencho CG. Sweet potatoes. In: SINHA NK, editor. Handbook of vegetables and vegetable processing. New Jersey: Wiley-Blackwell; 2011. p. 717-37.

2. Bouwkamp JC. Introduction-part 1. In: Bouwkamp JC, editor. Sweet potato products: a natural resource for the tropics. Boca Raton: CRC Press; 1985. p. 3-7.

3. Zörb C, Geilfus CM, Dietz KJ. Salinity and crop yield. Plant Biol German Soc Plant Sci. 2019;21:31-8

4. Majeed A, Muhammad Z. Salinity: a major agricultural problem—causes, impacts on crop productivity and management strategies. In: Hasanuzzaman M, Hakeem K, Nahar K, Alharby H, editors. Plant abiotic stress tolerance. Cham: Springer; 2019. p. 83-99.

5. Bhadauria V. Next-generation sequencing and bioinformatics for plant science. Wymondham: Caister Academic Press; 2017

6. Tao X, Gu Y, Wang H, Zheng W, Li X, Zhao C, Zhang Y. Digital gene expression analysis based on integrated de novo transcriptome assembly of sweet potato [lpomoea batatas (L.) Lam.]. PLoS ONE. 2012;7(4):e36234.

7. Lin Y, Zou W, Lin S, Onofua D, Yang Z, Chen H, Wang S, Chen XJP. Transcriptome profiling and digital gene expression analysis of sweet potato for the identification of putative genes involved in the defense response against Fusarium oxysporum f. sp. batatas. PLoS One. 2017;12(11):e0187838.

8. Li R, Zhai H, Kang C, Liu D, He S, Liu Q. De novo transcriptome sequencing of the orange-fleshed sweet potato and analysis of differentially expressed genes related to carotenoid biosynthesis. Int J Genomics. 2015:843802-12.

9. Loebenstein $\mathrm{G}$, Thottappilly G. Origin, distribution and economic importance. In: Loebenstein G, Thottappilly G, editors. The sweetpotato. Netherlands: Springer; 2009. p. 9-12.

10. Su X, Griffin J, Xu J, Ouyang P, Zhao Z, Wang W. Identification and quantification of anthocyanins in purple-fleshed sweet potato leaves. Heliyon. 2019;5(6):e01964.

11. Ma P, Bian X, Jia Z, Guo X, Xie Y. De novo sequencing and comprehensive analysis of the mutant transcriptome from purple sweet potato (Ipomoea batatas L.). Gene. 2016;575(2):641-9.

12. Xie F, Burklew CE, Yang Y, Liu M, Xiao P, Zhang B, Qiu D. De novo sequencing and a comprehensive analysis of purple sweet potato (Impomoea batatas L.) transcriptome. Planta. 2012;236(1):101-13.

13. Kumpatla SP, Buyyarapu R, Abdurakhmonov IY, Mammadov JA. Genomicsassisted plant breeding in the 21st century: technological advances and progress. In: Abdurakhmonov IY, editor. Plant Breeding, InTech; 2012. p. 131-84.

14. Gupta B, Huang B. Mechanism of salinity tolerance in plants: physiological biochemical, and molecular characterization. Int J Genomics. 2014;V2014: 701596-614.

15. Sunkar R. Plant stress tolerance. Methods Mol Biol. 2010;639:401.

16. Nong $Q$, Zhang $M$, Chen J, Zhang $M$, Cheng H, Jian S, Lu H, Xia K. RNA-Seq De Novo Assembly of Red Pitaya (Hylocereus polyrhizus) Roots and Differential Transcriptome Analysis in Response to Salt Stress. Trop Plant Biol. 2019:12(2):55-66.

17. Deng S, Ma J, Zhang L, Chen F, Sang Z, Jia Z, Ma L. De novo transcriptome sequencing and gene expression profiling of Magnolia wufengensis in response to cold stress. BMC Plant Biol. 2019;19(1):321.

18. Parchman TL, Geist KS, Grahnen JA, Benkman CW, Buerkle CA Transcriptome sequencing in an ecologically important tree species: assembly, annotation, and marker discovery. BMC Genomics. 2010;11(1):180.

19. Zhou A, Ma H, Liu E, Jiang T, Feng S, Gong S, Wang J. Transcriptome sequencing of Dianthus spiculifolius and analysis of the genes involved in responses to combined cold and drought stress. Int J Mol Sci. 2017; 18(4):849

20. Luo Y, Reid R, Freese D, Li C, Watkins J, Shi H, Zhang H, Loraine A, Song B-H. Salt tolerance response revealed by RNA-Seq in a diploid halophytic wild relative of sweet potato. Sci Rep. 2017;7(1):9624

21. Solis J, Baisakh N, Brandt SR, Villordon A, La Bonte D. Transcriptome Profiling of Beach Morning Glory (Ipomoea imperati) under Salinity and Its Comparative Analysis with Sweetpotato. PLoS ONE. 2016;11(2):e0147398. 
22. Luo Q, Teng W, Fang S, Li H, Li B, Chu J, Li Z, Zheng Q. Transcriptome analysis of salt-stress response in three seedling tissues of common wheat. Crop J. 2019;7(3):378-92.

23. Alberts B, Bray D, Hopkin K, Johnson AD, Lewis J, Raff M, Roberts K, Walter P. Essential cell biology: Garland Science; CRC Press; 2013. https://books. google.com.eg/books?id=Cg4WAgAAQBAJ.

24. Mahajan S, Tuteja N. Cold, salinity and drought stresses: An overview. Arch Biochem Biophys. 2005:444(2):139-58.

25. Murata N, Takahashi S, Nishiyama Y, Allakhverdiev SI. Photoinhibition of photosystem II under environmental stress. Biochim Biophys Acta (BBA) Bioenerg. 2007;1767(6):414-21.

26. Mittler R, Vanderauwera S, Gollery M, Van Breusegem F. Reactive oxygen gene network of plants. Trends Plant Sci. 2004;9(10):490-8.

27. Parida AK, Das AB. Salt tolerance and salinity effects on plants: a review. Ecotoxicol Environ Saf. 2005;60(3):324-49.

28. Botella MA, Rosado A, Bressan RA, Hasegawa PM. Plant adaptive responses to salinity stress. Plant Abiotic Stress. 2005;21:38-70.

29. Wang W, Vinocur B, Shoseyov O, Altman AJT. Role of plant heat-shock proteins and molecular chaperones in the abiotic stress response. Trends Plant Sci. 2004;9(5):244-52.

30. Wang W, Vinocur B, Altman AJP. Plant responses to drought, salinity and extreme temperatures: towards genetic engineering for stress tolerance. Planta. 2003;218(1):1-14.

31. Zhu J-K. Abiotic stress signaling and responses in plants. Cell. 2016;167(2):313-24.

32. Zhu J-K. Salt and drought stress signal transduction in plants. Annu Rev Plant Biol. 2002;53(1):247-73.

33. Deinlein U, Stephan AB, Horie T, Luo W, Xu G, Schroeder II. Plant salttolerance mechanisms. Trends Plant Sci. 2014;19(6):371-9.

34. Lata C, Yadav A, Prasad M. Role of plant transcription factors in abiotic stress tolerance. Abiotic Stress Response Plants Physiol Biochem Genet Perspect. 2011;10:269-96. https://doi.org/10.5772/23172 Arun Shanker and B. Venkateswarlu, INTECH Open Access Publishers.

35. Yang Z, Wang X, Gu S, Hu Z, Xu H, Xu C. Comparative study of SBP-box gene family in Arabidopsis and rice. Gene. 2008:407(1-2):1-11.

36. Mayer MP, Bukau B. Hsp70 chaperones: cellular functions and molecular mechanism. Cell Mol Life Sci. 2005;62(6):670-84.

37. Hothorn M, Wolf S, Aloy P, Greiner S, Scheffzek K. Structural Insights into the Target Specificity of Plant Invertase and Pectin Methylesterase Inhibitory Proteins. Plant Cell. 2004;16(12):3437-47.

38. Negrao S, Schmöckel S, Tester M. Evaluating physiological responses of plants to salinity stress. Ann Bot. 2016;119(1):1-11.

39. Geng Y, Wu R, Wee CW, Xie F, Wei X, Chan PMY, Tham C, Duan L, Dinneny JR. A spatio-temporal understanding of growth regulation during the salt stress response in Arabidopsis. Plant Cell. 2013:25(6):2132-54.

40. Guex N, Henry H, Flach J, Richter H, Widmer F. Glyoxysomal malate dehydrogenase and malate synthase from soybean cotyledons (Glycine max L.): enzyme association, antibody production and CDNA cloning. Planta. 1995;197(2):369-75.

41. Halford Nigel G, Hey Sandra J. Snf1-related protein kinases (SnRKs) act within an intricate network that links metabolic and stress signalling in plants. Biochem J. 2009;419(2):247-59.

42. Chen J, Gao L, Baek D, Liu C, Ruan Y, Shi H. Detoxification function of the Arabidopsis sulphotransferase AtSOT12 by sulphonation of xenobiotics. Plant Cell Environ. 2015;38(8):1673-82.

43. Cushman JC, Bohnert HJ. Genomic approaches to plant stress tolerance. Curr Opin Plant Biol. 2000;3(2):117-24.

44. Hussain S, Zhu C, Bai Z, Huang J, Zhu L, Cao X, Nanda S. iTRAQ-Based Protein Profiling and Biochemical Analysis of Two Contrasting Rice Genotypes Revealed Their Differential Responses to Salt Stress. Int J Mol Sci. 2019;20(3):547.

45. Marrocco K, Zhou Y, Bury E, Dieterle M, Funk M, Genschik P, Krenz M, Stolpe T, Kretsch T. Functional analysis of EID1, an F-box protein involved in phytochrome A-dependent light signal transduction. Plant J Cell Mol Biol. 2006;45(3):423-38.

46. Hartmann L, Pedrotti L, Weiste C, Fekete A, Schierstaedt J, Göttler J, Kempa S, Krischke M, Dietrich K, Mueller MJ, et al. Crosstalk between Two bZIP Signaling Pathways Orchestrates Salt-Induced Metabolic Reprogramming in Arabidopsis Roots. Plant Cell. 2015;27(8):2244-60

47. Kazanietz MG, Wang S, Milne GW, Lewin NE, Liu HL, Blumberg PM. Residues in the second cysteine-rich region of protein kinase $\mathrm{C}$ delta relevant to phorbol ester binding as revealed by site-directed mutagenesis. J Biol Chem. 1995;270(37):21852-9.

48. Idänheimo N, Gauthier A, Salojärvi J, Siligato R, Brosché M, Kollist H, Mähönen AP, Kangasjärvi J, Wrzaczek M. The Arabidopsis thaliana cysteinerich receptor-like kinases CRK6 and CRK7 protect against apoplastic oxidative stress. Biochem Biophys Res Commun. 2014;445(2):457-62.

49. Meng K, Yun-gang Z, Ya-ju L, Xin W, Wei T, Hui Y, Dai-fu M, Qiang L. Breeding and characterization of a New Sweetpotato Cultivar Xuzi 8 with High Anthocyanin Content for Table Use and Processing. Southwest China J Agric Sci. 2019;6(27):633-9.

50. Arisha MH, Qiang YQ, Fei GR, Hui Y, Meng K, Wei T, Ju LY, Xin W, Gang ZY, Qiang L. Stress signals determination in different sweet potato cultivars at seedling stage under salt stress conditions. Zagazig J Agric. 2019;4(1):150-62.

51. Grabherr MG, Haas BJ, Yassour M, Levin IZ, Thompson DA, Amit I, Adiconis X, Fan L, Raychowdhury R. Zeng QJNb: Full-length transcriptome assembly from RNA-Seq data without a reference genome. Nat Biotechnol. 2011;29(7):644.

52. Haas BJ, Papanicolaou A, Yassour M, Grabherr M, Blood PD, Bowden J, Couger MB, Eccles D, Li B. Lieber MJNp: De novo transcript sequence reconstruction from RNA-seq using the Trinity platform for reference generation and analysis. Nat Protoc. 2013;8(8):1494.

53. Götz S, García-Gómez JM, Terol J, Williams TD, Nagaraj SH, Nueda MJ, Robles M, Talón M, Dopazo J. Conesa AJNar: High-throughput functional annotation and data mining with the Blast2GO suite. Nucleic Acids Res. 2008;36(10):3420-35.

54. Young MD, Wakefield MJ, Smyth GK. Oshlack AJGb: Gene ontology analysis for RNA-seq: accounting for selection bias. Genome Biol. 2010;11(2):R14.

55. Mao X, Cai T, Olyarchuk JG, Wei LB. Automated genome annotation and pathway identification using the KEGG Orthology (KO) as a controlled vocabulary. Bioinformatics. 2005;21(19):3787-93.

56. Li B. Dewey CNJBb: RSEM: accurate transcript quantification from RNA-Seq data with or without a reference genome. BMC Bioinformatics. 2011;12(1):323.

57. Storey JD. Tibshirani RJPotNAoS: Statistical significance for genomewide studies. Proc Natl Acad Sci. 2003;100(16):9440-5.

58. Guo M, Liu J-H, Ma X, Zhai Y-F, Gong Z-H, Lu M-H. Genome-wide analysis of the $\mathrm{Hsp} 70$ family genes in pepper (Capsicum annuum L.) and functional identification of CaHsp70-2 involvement in heat stress. Plant Sci. 2016;252:246-56.

59. Wan H, Yuan W, Ruan M, Ye Q, Wang R, Li Z, Zhou G, Yao Z, Zhao J, Liu S Identification of reference genes for reverse transcription quantitative realtime PCR normalization in pepper (Capsicum annuum L.). Biochem Biophys Res Commun. 2011;416(1-2):24-30.

60. Livak KJ, Schmittgen TD. Analysis of relative gene expression data using real-time quantitative PCR and the $2-\Delta \Delta C T$ method. Methods. 2001;25(4): $402-8$.

\section{Publisher's Note}

Springer Nature remains neutral with regard to jurisdictional claims in published maps and institutional affiliations.

Ready to submit your research? Choose BMC and benefit from:

- fast, convenient online submission

- thorough peer review by experienced researchers in your field

- rapid publication on acceptance

- support for research data, including large and complex data types

- gold Open Access which fosters wider collaboration and increased citations

- maximum visibility for your research: over $100 \mathrm{M}$ website views per year

At $\mathrm{BMC}$, research is always in progress.

Learn more biomedcentral.com/submissions 